\title{
Quality Assessment and Circularity Potential of Recovery Systems for Household
} Plastic Waste

\author{
Eriksen, Marie Kampmann; Damgaard, Anders; Boldrin, Alessio; Astrup, Thomas Fruergaard
}

Published in:

Journal of Industrial Ecology

Link to article, DOI:

$10.1111 /$ jiec. 12822

Publication date:

2019

Document Version

Peer reviewed version

Link back to DTU Orbit

Citation (APA):

Eriksen, M. K., Damgaard, A., Boldrin, A., \& Astrup, T. F. (2019). Quality Assessment and Circularity Potential of Recovery Systems for Household Plastic Waste. Journal of Industrial Ecology, 23(1), 156-168.

https://doi.org/10.1111/jiec.12822

\section{General rights}

Copyright and moral rights for the publications made accessible in the public portal are retained by the authors and/or other copyright owners and it is a condition of accessing publications that users recognise and abide by the legal requirements associated with these rights.

- Users may download and print one copy of any publication from the public portal for the purpose of private study or research.

- You may not further distribute the material or use it for any profit-making activity or commercial gain

- You may freely distribute the URL identifying the publication in the public portal 
9 Address correspondence to:

10 Marie Kampmann Eriksen

11 Technical University of Denmark

12 Bygningstorvet 115, DK-2800 Kgs. Lyngby, Denmark

13 maker@env.dtu.dk

14

15 Conflict of interest: Nothing to declare

16 


\section{$<$ heading level 1 > Summary}

Plastic recycling is promoted in the transition towards circular economy and a closed plastic loop, typically using mass-based recycling targets. Plastic from household waste (HHW) is contaminated and heterogeneous, and recycled plastic from HHW often has a limited application range, due to reduced quality. To correctly assess the ability to close plastic loops via recycling, both plastic quantities and qualities need to be evaluated. This study defines a circularity potential representing the ability of a recovery system to close material loops assuming steady-state market conditions. Based on an average plastic waste composition including impurities, 84 recovery scenarios representing a wide range of sorting schemes, source-separation efficiencies and material recovery facility (MRF) configurations and performances were assessed. The qualities of the recovered fractions were assessed based on contamination, and the circularity potential calculated for each scenario in a European context. Across all scenarios, $17-100 \%$ of the plastic could be recovered, with higher source-separation and MRF efficiencies leading to higher recovery. Including quality, however, at best $55 \%$ of the generated plastic was suitable for recycling due to contamination. Source-separation, a high number of target fractions and efficient MRF recovery were found critical. The circularity potential illustrated that less than $42 \%$ of the plastic loop can be closed with current technology and raw material demands. Hence, Europe is still far from able to close the plastic loop. When transitioning towards circular economy, focus should be on limiting impurities and losses, through product design, technology improvement and more targeted plastic waste management.

Keywords: circular economy, contamination, post-consumer waste, substitution, life cycle assessment 


\section{$<$ heading level 1 $>$ Introduction}

Circular economy concepts have gained increasing attention in recent decades as an approach to overcome both economic and environmental challenges. One of the environmental challenges is to minimize material loss and reduce pressure on primary resources, by transitioning from the linear material consumption in current systems to closed material loops in a circular economy (EMF, 2016; Braungart and McDonough, 2002; EC, 2015). Recycling of materials is crucial in this transition and recently the European Union has defined new mass-based recycling targets, as part of the European circular economy strategy, namely $60 \%$ and $65 \%$ recycling of municipal solid waste (MSW) in 2030 and 2035, respectively (EC, 2018a). Such targets focus on waste quantities routed to recycling rather than the actual amounts of recovered materials being recycled, the quality of the recycled materials and the substitution of virgin raw materials (EC, 2018a). However, MSW and especially household waste (HHW) represent highly heterogeneous material streams; even individual recovered material fractions are heterogeneous and contains a variety of impurities in addition to the target material itself (Heinzel et al., 2015), influencing the quality. Consequently, the potential for recycled materials from HHW to substitute virgin materials depends not only on the quantities but particularly on the quality of the waste materials and their ability to fulfill the functionality of the raw materials substituted (Vadenbo et al., 2016). As "low-quality" recovered waste materials with limited applicability cannot substitute "highquality" virgin materials with a wider application range, the functionality of the two materials is not compatible. In a theoretical end-point goal of closed material loops, the potential of a recycling system to close material loops will therefore depend on the ability of the system to provide material quantities and qualities fulfilling the demands in a steady-state market. Thus, to better evaluate how recycling systems contribute to closing of material loops, we need to look beyond mass-based recycling rates and traditional substitution ratios and instead address the potential contribution to "material circularity" of recovery and recycling systems, for which the quality of the recycled materials is crucial.

Recycling of plastic is a prominent example of a material for which quality is critical. Plastic plays a key role within circular economy with high regulatory recycling targets; for example, the EU has proposed a recycling rate of $55 \%$ for plastic packaging waste by 2030 , placing specific emphasis on plastic in HHW (EC, 2018b; EC, 2018c). Plastic from HHW is a particularly heterogeneous waste stream 
containing both high-quality items, such as food contact-approved plastic, and lower-quality items, such as flower pots and dirty non-food containers (Petersen et al., 2012; Petersen et al., 2014). From a recycling perspective, the quality of plastic waste is affected mainly by the contamination level of the recovered plastic (Ragaert et al., 2017; van der Harst et al., 2016; Villanueva and Eder, 2014), which can be divided into four main groups: 1) the presence of non-plastic items, e.g. missorted items, composite materials, poor cleaning, 2) the presence of non-targeted polymer types, e.g. from items containing several polymers, labels, multi-layer plastic films or mis-sorting, 3) the presence of unwanted product types, e.g. toys, if bottles are the targeted product category, and 4) chemical contamination, e.g. from colorants, stabilizers, compatibilizers, use or waste management (Dahlbo et al., 2017). Although the quality of recycled plastic are affected directly by these physical and chemical properties, only few studies have quantitatively addressed plastic quality based on these (e.g. Huysman et al., 2017).

To fully close plastic polymer loops, recovered plastic materials need to be recycled into new products at the same or similar quality levels as the original plastic product, i.e. within applications comparable to the original products. However, recycling of higher-quality plastic into lower-quality application levels is a well-known challenge, often termed "downcycling", involving considerable losses of material properties compared to virgin plastic (Rigamonti et al., 2018; van der Harst et al., 2016; Luijsterburg and Goossens, 2014; Vilaplana and Karlsson, 2008), reducing the quality and thereby applicability of the recycled plastic. Various attempts have been made in life cycle assessment (LCA) studies to include such quality losses of recycled plastic in the estimation of the substitutability (also called substitution ratio, substitution factor, etc.), defined as the functionality of the recycled plastic divided by the functionality of the virgin plastic assumed substituted (Vadenbo et al., 2016). In these studies the functionality or quality was quantified based on e.g. price differences between recovered and virgin materials (e.g. Rigamonti et al., 2009; Mengarelli et al., 2017), practical experiences in the recycling industry (Gu et al., 2017), or qualitative discussions (Shen et al., 2010). While these approaches attempt to quantify loss of material quantities as well as physical and mechanical properties, such as higher thickness required, more defects, lower transparency, etc., substitutabilities such as these are not useful for evaluating the ability of a recovery or recycling system to contribute to long-term closing of material loops. 
As the current European market for recyclable polymers by far is saturated (Fellner et al., 2017), recovered plastic waste may be fully recycled and substituted according to substitution ratios reflecting material and property losses, market responses, etc. (as indicated above). In other words, the substitution of recovered plastic waste may be "high", as the current polymer market can fully absorb the low-quality plastic waste, even if the quality of the recovered plastic and thereby the substitutability in long-term steady-state conditions is "low" due to the presence of impurities. Consequently, the abilities of such low-quality recycled plastic to close the plastic loop in a long-term perspective are small as such qualities only have the potential to substitute virgin plastic in parts of the market and thereby do not have the potential to close the part of the loop relying on higher quality material. We therefore suggest extending the existing definitions of substitutability and substitution ratios to more appropriately reflect the potential of a recovery system to contribute to long-term closing of material loops, i.e. the "circularity potential" of a recovery or recycling system. This should be understood as a supplement to existing substitution ratios typically applied in current LCA studies of recycling. So far no attempts have been provided to systematically assess and quantify this circularity and evaluate the associated importance of quantity and quality of recovered plastic from HHW.

The overall aim of this study was to define a "circularity potential" reflecting the ability to close material loops and apply this concept to a range of illustrative plastic recovery systems based on information about quantities and qualities of the recovered plastic. This was achieved by evaluating 84 hypothetical plastic recovery scenarios involving household waste (HHW) and determining the circularity potential for these scenarios. The following specific objectives were addressed: 1) application of material flow analysis (MFA) on selected plastic recovery scenarios, covering a wide range of sorting schemes, source-separation efficiencies, material recovery facility (MRF) efficiencies and configurations with the purpose of estimating mass-based losses in the system until reprocessing, 2) assessment of the potential quality of all MRF outputs in the scenarios according to the level of contamination, 3) development of the circularity potential by extending existing definitions of substitutability and involving information about market shares in a potential steady-state situation with closed material loops, and 4) evaluating the implications of the circularity potential for waste recycling assessment and the transition towards circular economy. 
A generic European waste composition was assumed for all scenarios, including all waste generated in the households, both plastic and non-plastic material fractions. The share of plastic in the waste was assumed to be $14 \%$ by weight, while the remaining $86 \%$ was assumed to be non-plastic (Edjabou et al., 2015). Table 1 provides an overview of the included plastic fractions and associated polymer types. Focus was on the most abundant polymer types in European HHW, i.e. polyethylene terephthalate (PET), high density polyethylene (HDPE), low density polyethylene (LDPE), polypropylene (PP), and polystyrene (PS) (Götze and Astrup, 2013), while the remaining plastic was categorized as "Others". The fractional composition of plastic waste was estimated based on Rigamonti et al. (2014), Petersen et al. (2015) and Edjabou et al. (2015).

Table 1 Composition of the plastic part of the HHW divided into plastic fractions and polymer types [\%]. The composition was estimated based on Rigamonti et al. (2014), Edjabou et al. (2015) and Petersen et al. (2015).

\begin{tabular}{lcccccc|c}
\hline Plastic fractions & PET & HDPE & LDPE & PP & PS & Others & Total \\
\hline Bottles & $23 \%$ & $7 \%$ & $0 \%$ & $0 \%$ & $0 \%$ & $0 \%$ & $30 \%$ \\
Soft packaging & $0 \%$ & $0 \%$ & $30 \%$ & $0 \%$ & $0 \%$ & $10 \%$ & $40 \%$ \\
Hard packaging & $4 \%$ & $3 \%$ & $0 \%$ & $7 \%$ & $1 \%$ & $5 \%$ & $20 \%$ \\
Other plastic items & $0 \%$ & $0 \%$ & $0 \%$ & $0 \%$ & $0 \%$ & $10 \%$ & $10 \%$ \\
\hline Total & $26 \%$ & $10 \%$ & $30 \%$ & $7 \%$ & $1 \%$ & $25 \%$ & $100 \%$ \\
\hline a) 23 and 4 are rounded and thus sum to 26 & & &
\end{tabular}
separated from the plastic waste during source-separation and subsequent sorting (figure 1), hence leaving the system. However, the remaining part of the non-plastic fractions ended up as non-plastic impurities in the plastic outputs from the MRF, thereby contaminating these outputs. The degree of contamination depended on the specific scenario. 
148 In total, 84 plastic recovery scenarios were defined as a combination of choices in four steps of the 149 recovery-chain, as illustrated in figure 1:

150

1a. Selection of target fractions and overall sorting scheme (A to F)

1b. Source-separation and collection efficiency (low/high)

2. MRF performance, modeled as the recovery efficiencies of target materials to intended outputs (low/average/high)

3. Numbers and types of outputs recovered from the MRF. combinations that were deemed unrealistic 84 scenarios remained (see details in the following and in the supporting information (SI), section S1). The amount of recovered plastic was determined based on a material flow analysis (MFA) model for each scenario, using the ingoing waste composition presented in table 1 as well as the source-separation and MRF efficiencies presented in the following sections. The potential quality of the plastic for recycling was determined based on the composition of the recovered plastic output from the MRF (see later sections for details), i.e. before reprocessing of the plastic. The reason for this was twofold: 1) existing quality criteria for recyclable plastic precisely addresses this point in the value-chain (see details in the SI, section S2.2), and 2) future recycling targets in EU are calculated based on waste input quantities to the "final recycling process" (EC, 2015). The system boundaries thereby reflect these perspectives, albeit excluding potential further losses in reprocessing. Figure 1 indicates the system boundaries. 


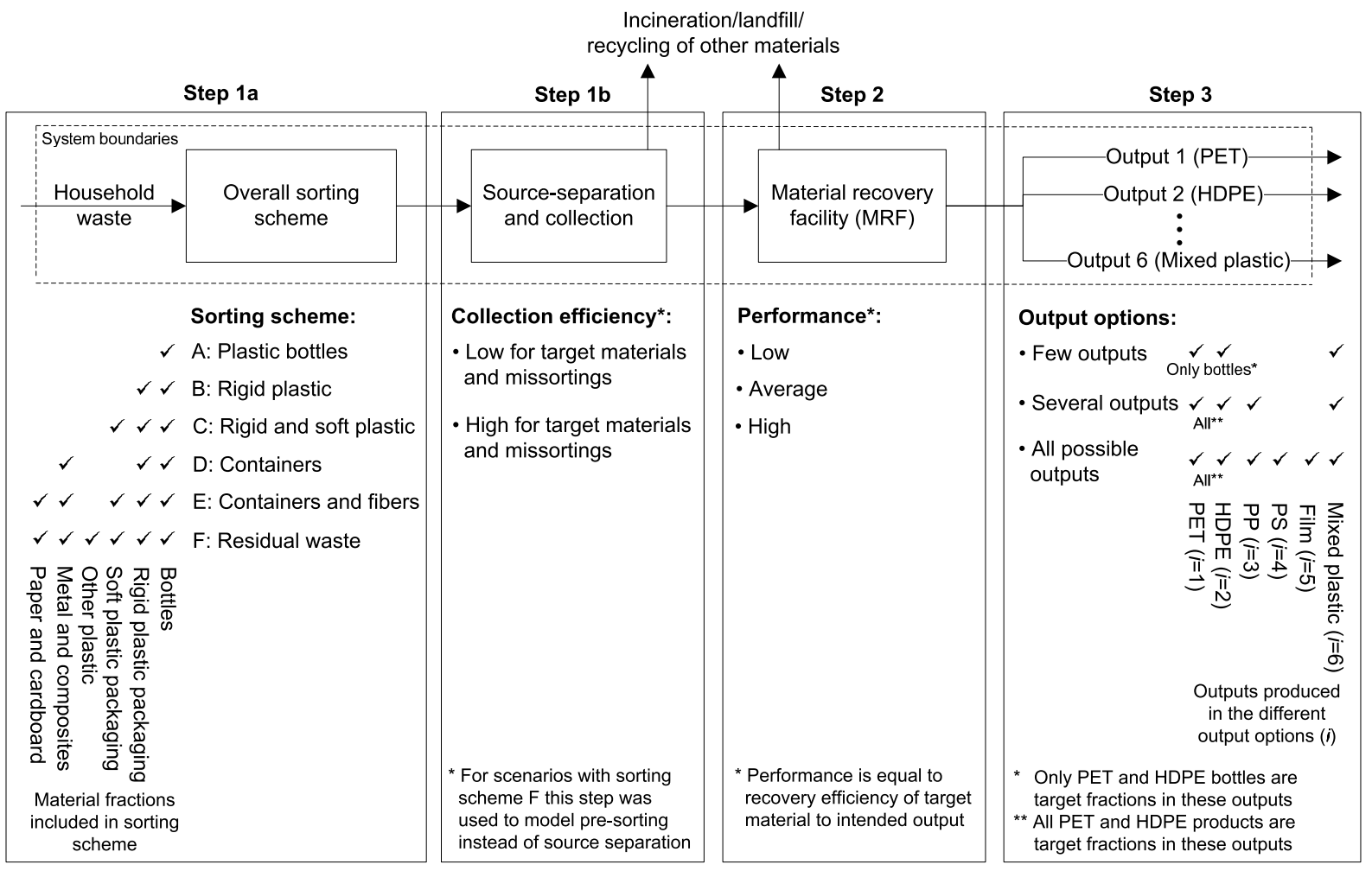

171 Figure 1 Possible scenario configurations. The different choices for each step are stated below the step.

172 Material fractions included in the overall sorting scheme are indicated (step 1a), as well as targeted

173 fractions in the different output choices (step 3). Containers refer to plastic, metal, and composite containers. $i$ refers to eq. (1) and (2).

Within each step, selections were made with the intention of defining a range of key types of recovery pathways representing differences in approaches to sorting, collecting, and recovering of plastic waste. Steps 1a and 3 represented system configurations selected to illustrate important combinations rather than provide an exhaustive list of all possible configurations. Step $1 \mathrm{~b}$ and 2 represented different efficiencies in source-separation and MRF performance; values reported in literature were used to define minimum, average and maximum efficiencies. The use of minimum and maximum values as well as the wide range of system configurations represent a scenario-based approach to account for the considerable variations in facility performance as well as data scarcity and uncertainty.

184 A full list of scenarios is given in the SI, tables S4 and S5, and a detailed description of all scenarios is provided in the SI, section S1. 
188 The first five sorting schemes (A to E) represent source-separation of plastics in the household, either 189 by targeting plastic fractions only (A: Plastic bottles, B: Rigid plastic, C: Rigid and soft plastic) or 190 through comingled separation schemes where plastic is collected with other materials (D: Containers,

191 E: Containers and Fibers). The last sorting scheme (F: No source-separation) represents a situation with 192 no source-separation, where all residual waste is routed to a sorting facility, sometimes called a "dirty 193 MRF" or a "mixed waste MRF" (e.g. Cimpan et al., 2015; Pressley et al., 2015). Several studies have 194 shown that the share of impurities and missorted items entering the MRF is higher in comingled systems 195 compared to source-separated plastic (Papineschi et al., 2016; Cappadona, 2015; Seyring et al., 2015; 196 Heinzel et al., 2015), and it was therefore assumed that increasing levels of impurities and missorted items was collected with the plastic, as the number of targeted fractions increased. For the "dirty MRF" system, a pre-sorting step, where the residual waste is coarsely sorted into different material streams including a "primary plastic flow", was considered necessary in order to increase the share of plastic in the waste stream entering the actual plastic sorting step (step 2) (Feil et al., 2016). Thus for sorting scheme F step $1 \mathrm{~b}$ was used to model such mechanical pre-sorting, instead of source-separation and collection. Table 2 provides an overview of the share of missorted items assumed for the individual scenarios in the cases of low and high source-separation efficiencies. For sorting scheme F, data in the literature did not warrant distinguishing between low and high efficiencies. More details are given in the SI, section S1.2.

Table 2: Missorted items in the plastic waste after source-separation and collection (pre-sorting for sorting scheme F) [\%], depending on the overall sorting scheme.

\section{Overall sorting scheme}

A: Plastic bottles

B: Rigid plastic

C: Rigid and soft plastic

D: Containers

E: Containers and fibers

F: No source-separation
Missorted items in the plastic waste sent to mechanical sorting Low source-separation efficiencies

\section{$2 \%$}

$4 \%$

$5 \%$

$9 \%$

$14 \%$

efficiencies

$4 \%$

$5 \%$

$11 \%$

$16 \%$ 
$<$ heading level 3> Source-separation efficiencies (step 1b) and MRF performance (step 2)

212 Source-separation efficiencies and MRF recovery efficiencies are presented in table 3 and table 4,

213 respectively, and are based on the range of efficiencies reported in literature (see details in the SI, section

214 S1.2 and S1.3). The MRF performance was modeled as the recovery efficiency of a target plastic fraction

215 directed to the intended output for that specific fraction, i.e. MRF performance was high if the recovery

216 efficiency of PET bottles directed to the PET output was high. As MRFs can vary considerably in

217 performance, due to great differences in configuration of equipment and manual sorting, three selections

218 were modeled: high, average and low. The recovery efficiency for the target fractions were set to the

219 highest ones reported in literature for the high performing MRFs and the lowest for the low performing

220 MRFs, whereas the recovery efficiencies in the average scenarios were always an average of the high

221 and low ones. This was also the case for the recovery efficiencies for the non-targeted fractions, which

222 were estimated since no data was available (details in SI, section S1.3). Based on a study reporting that

$22374-82 \%$ of plastic from residual waste was heavily contaminated by the remaining waste (Petersen and

224 Mayland, 2015), scenarios with sorting scheme F were assumed only to have low and medium

225 performing MRFs, as MRF recovery efficiencies are affected by surface contamination. Most of the

226 plastic not ending in the output for which they were intended ended in the mixed plastic fraction, which was produced in all scenarios. Hence, the lower the MRF performance, the more plastic ends in the mixed plastic fraction and vice versa. All recovery efficiencies, for both targeted fractions (e.g. PET bottles to PET output) and non-targeted fractions (e.g. PET bottles to HDPE output) to all outputs, are presented in the SI, table S1-S3. 
Table 3: High and low source-separation efficiencies [\%] of target fractions (TF) and non-target

236 fractions $(\mathrm{N}-\mathrm{TF})$.

\begin{tabular}{|c|c|c|c|c|}
\hline \multirow{2}{*}{ Material fractions } & \multirow{2}{*}{\multicolumn{2}{|c|}{$\begin{array}{c}\text { Low source-separation efficiencies } \\
\text { TF }\end{array}$}} & \multicolumn{2}{|c|}{ High source-separation efficiencies } \\
\hline & & & TF & N-TF \\
\hline Bottles $^{1}$ & $65 \%$ & - & $90 \%{ }^{4}$ & - \\
\hline Rigid packaging & $30 \%$ & $10 \%$ & $60 \%$ & $20 \%$ \\
\hline Foil packaging & $30 \%$ & $10 \%$ & $60 \%$ & $20 \%$ \\
\hline Other plastic items ${ }^{2}$ & - & $10 \%$ & - & $20 \%$ \\
\hline Non-plastic items $s^{2,3}$ & - & $0.001-0.01 \%$ & - & $0.002-0.02 \%$ \\
\hline
\end{tabular}

1) Targeted within all the separation schemes.

2) Not targeted within any of the separation schemes.

3) Vary depending on the overall sorting scheme, see table 2.

4) Currently only reached through refund deposit systems

Table 4: Recovery efficiencies [\%] of target material fractions to target MRF outputs (e.g. PET bottles

to PET output or rigid PP to PP output), depending on MRF performance.

\begin{tabular}{l|cc|cc|cc|cc|c}
\hline \multirow{2}{*}{} & \multicolumn{2}{|c|}{ PET } & \multicolumn{2}{c|}{ HDPE } & \multicolumn{2}{c|}{ PP } & \multicolumn{2}{c|}{ PS } & LDPE \\
MRF performance & Bottles & Rigid & Bottles & Rigid & Bottles & Rigid & Bottles & Rigid & Film \\
\hline Low & $40 \%$ & $20 \%$ & $35 \%$ & $15 \%$ & $25 \%$ & $25 \%$ & $25 \%$ & $25 \%$ & $10 \%$ \\
Average & $68 \%$ & $55 \%$ & $63 \%$ & $50 \%$ & $55 \%$ & $55 \%$ & $48 \%$ & $48 \%$ & $53 \%$ \\
High & $95 \%$ & $90 \%$ & $90 \%$ & $85 \%$ & $85 \%$ & $85 \%$ & $70 \%$ & $70 \%$ & $95 \%$ \\
\hline
\end{tabular}

$<$ heading level 3> MRF outputs (step 3)

242 Three different MRF output choices were considered: Few, several, or all possible outputs. Figure 1

243 illustrates the specific types of outputs produced in the three output choices. All three output choices are possible for the sorting schemes B-F. For scenarios employing sorting scheme A (only targeting bottles in the overall separation scheme), the "few MRF output" choice was the only realistic option, since very little PP, PS, or film was present in the collected waste stream. 


\section{$<$ heading level 2> Resource recovery efficiency, $\eta^{\text {rec }}$}

253 The physical losses in each scenario were identified through the resource recovery efficiency, $\eta^{\text {rec }}$, as 254 defined by Vadenbo et al. (2016), presented in eq. (1):

$$
\eta^{r e c}=\frac{\sum_{i=1}^{6} M_{i}^{r e c}}{U^{r e c}}
$$

255

256

- $\quad \eta^{\text {rec }}[-]$ is the resource recovery efficiency, including all physical material losses within the recycling chain. As the system boundaries for this study do not include the reprocessing facility, $\eta^{\text {rec }}$ is the product of source-separation efficiencies and MRF recovery efficiencies.

- $U^{\text {rec }}[\mathrm{kg}]$ is the resource potential of recovered material and expresses the amount of target material in the waste stream under assessment, e.g. the amount of plastic in the HHW. $U^{\text {rec }}$ was set to $1 \mathrm{~kg}$, corresponding to $100 \%$ of the generated plastic waste (table 1 ).

- $\quad M^{r e c}[\mathrm{~kg}]$, is the amount of material in each individual output, $i$, recovered from the MRF. $M^{\text {rec }}$ was defined both with and without impurities, as described in the following.

- $i$ represents the individual outputs recovered from the MRF (1=PET, 2=HDPE, $3=$ PP, 4=PS, 5=film, $6=\operatorname{mix})$.

To clearly illustrate the importance of impurities in the different MRF outputs, four types of $\eta^{\text {rec }}$ were calculated for each scenario, depending on the definition of $M^{\text {rec }}$ and $i$ :

1. All MRF outputs - with impurities $\left(i=1-6, M^{\text {rec }}=\right.$ mass of all material recovered in the individual outputs)

2. All MRF outputs - without impurities $\left(i=1-6, M^{\text {rec }}=\right.$ mass of target polymer recovered in the individual MRF outputs. e.g. $M_{P P}^{r e c}$ only included the mass of recovered PP plastic in the PP output)

3. Mono-polymer outputs (PET, HDPE, PP and PS) - with impurities $\left(i=1-4, M^{\text {rec }}=\right.$ mass of all material recovered in the individual outputs)

4. Mono-polymer outputs - without impurities $\left(i=1-4, M^{\text {rec }}=\right.$ mass of target polymer recovered in the individual MRF outputs) 
279 The quality of plastic for recycling depends on a wide range of properties such as physical and chemical

280 composition, mechanical strength, color, odor, additive concentration, and content of toxic chemicals.

281 As such, a single and unique parameter cannot be applied to represent the quality for all possible application types. Acknowledging this, the approach applied in this study to describe quality involved two steps: 1) identification of the most important application groups for plastic in Europe, and 2) classification of the MRF outputs according to the quality criteria available for these application groups. Consequently, the quality as defined here represents the potential applicability of the recovered plastic relative to the defined quality levels presented below. PlasticsEurope and EPRO (2016)), eight key application groups were identified. The application groups are listed below according to the strictness of the legal requirements to the chemical composition and/or migration behavior of the material.

○ Toys

- Pharmaceuticals

- Electrical and electronics

- Building and construction

○ Non-food packaging

○ Automotive

- Others 
for food contact, representing the strictest legal material requirements, 2) medium quality ( $Q$ = medium), assigned to materials that can be used for toys, pharmaceuticals and electrical and electronics, representing lower and varying, legal material requirements, and 3) low quality ( $Q=$ low), assigned to materials with minimal legal requirements.

Hence, high quality material is defined as material with the ability to fulfil all demands in the respective polymer market, i.e. for recovered polymers to be used in all eight application groups within the specific polymer market, and thereby having a potential to substitute virgin plastic in the entire polymer loop. Consequently, if recovered polymers have a medium or low quality, they comply only with a subset of available applications (i.e. seven for medium and four for low quality), and thereby only have the potential to close the polymer loops with respect to these applications, as the remaining applications will still have to rely on virgin material. Per definition, virgin plastic is considered high quality ( $Q=$ high) as the composition of virgin plastic can be controlled during production to match specifically the application in question. References to all relevant legislation as well as definition of application groups and quality levels are provided in the SI, sections S2.

All MRF outputs from the 84 scenarios were classified into one of the three quality levels. As very few studies and data related to chemical contamination of plastic waste exist (Pivnenko et al., 2016; Ballesteros-Gómez et al., 2014; Whitt et al., 2012; Riber et al., 2009; Ernst et al., 2000; Huber and Franz, 1997), including chemical contamination in the assessment was not feasible. Therefore, the classification was carried out based solely on the presence of physical impurities, including both nonplastic and non-target polymer impurities. All steps in the quality assessment are described in detail in the SI, section S2.2, including a summary of legal limit values (table S10), quality criteria defined by plastic reprocessing facilities (tables S7-S9), and a detailed description of how these criteria were applied in the classification. 
The circularity potential depends both on the level of physical losses in the system as well as the quality loss of the recycled plastic relative to the displaced virgin plastic. It is here suggested that the circularity potential [-], $c^{\text {rec }}$, can be defined as a function of the resource recovery efficiency of the system, $n^{\text {rec }}$, and the market share [-], MS, in which the materials with a specific quality level [-], $Q$, has a potential to be applied (and thereby substitute virgin material), as presented in eq. (2):

$$
c^{r e c}=\sum_{i=1}^{6} \eta_{i}^{r e c} \cdot \frac{M S\left(Q^{r e c}\right)_{i}}{M S\left(Q^{\text {disp }}\right)_{i}} \rightarrow\left\{\begin{array}{c}
M S_{\text {high }} \text { for } Q=\text { high }, \\
M S_{\text {medium }} \text { for } Q=\text { medium } \\
M S_{\text {low }} \text { for } Q=\text { low }
\end{array}\right.
$$

337

As described in the previous section the quality of the potentially displaced virgin material, $Q^{d i s p}$, is always assumed high and consequently $M S\left(Q^{d i s p}\right)$ is equal to 1 for all MRF outputs, $i$. The second multiplier of eq. (2) is analogue to the substitutability definition by Vadenbo et al. (2016) who divided the functionality of the recovered material with the functionality of the displaced material. In case of the circularity potential, assuming a theoretical end-point market situation with closed polymer loops under steady-state conditions, the functionality is represented by the fraction of the total polymer market within which the recovered plastic with a specific quality is applicable and can fulfill the material requirements (as described in the previous section). Thereby, the functionality now expresses the potential ability of a recovered material fraction to fulfill the demands in a steady-state market and contribute to a circular economy vision.

Market shares, $M S$, for low-, medium-, and high-quality plastic were defined for the European markets of PET, HDPE, PP, PS, film, and mixed plastic by combining information from PlasticEurope and EPRO (2016) with several other sources (for details see SI, section S3). Market shares are presented in Table 5 and were determined as relative shares of the European production in 2016 (mainly virgin production) for the individual application groups mentioned previously. As an example, recovered HDPE with a medium quality can substitute virgin HDPE in medium and low quality application groups, corresponding to a market share of $3 \%+70 \%=73 \%$. Consequently, $M S(\text { medium })_{H D P E}=0.73$, illustrating that recovered HDPE of medium quality has a theoretical potential to close $73 \%$ of the HDPE 
loop, assuming a steady-state HDPE market and no material loss. Although forecasted information for

357 potential future market situations could have been included, here the calculations were based on current market information to reflect the potential of the recovery scenarios (existing technologies) to close plastic loops in a situation with the current consumption.

Table 5: European market share of all application groups for the PET, HDPE, PP, PS, film, and mixed plastic European markets [\%], divided into the three quality levels. $M S$ values for use in eq. 2 [-] are provided for all $Q$.

\begin{tabular}{l|c|cccccc}
\hline \multirow{2}{*}{ Application groups } & \multirow{2}{*}{ Unit } & \multicolumn{6}{|c}{ European polymer markets $(i)$} \\
& & PET & HDPE & Film & PP & PS & Mix* \\
\hline High quality & $\%$ & $\mathbf{5 7}$ & $\mathbf{2 7}$ & $\mathbf{5 4}$ & $\mathbf{1 8}$ & $\mathbf{1 5}$ & $\mathbf{3 4}$ \\
Food packaging & $\%$ & 57 & 27 & 54 & 18 & 15 & 34 \\
\hline Medium quality & $\%$ & $\mathbf{0}$ & $\mathbf{3}$ & $\mathbf{4}$ & $\mathbf{6}$ & $\mathbf{1 1}$ & $\mathbf{6}$ \\
Toys & $\%$ & 0 & 0 & 0 & 0 & 0 & 1 \\
Pharmaceuticals & $\%$ & 0 & 1 & 1 & 1 & 1 & 1 \\
Electrical and electronics & $\%$ & 0 & 2 & 3 & 5 & 10 & 4 \\
\hline Low quality & $\%$ & $\mathbf{4 3}$ & $\mathbf{7 0}$ & $\mathbf{4 2}$ & $\mathbf{7 6}$ & $\mathbf{7 4}$ & $\mathbf{6 0}$ \\
Building and construction & $\%$ & 0 & 23 & 6 & 8 & 42 & 13 \\
Non-food packaging & $\%$ & 42 & 23 & 18 & 20 & 18 & 22 \\
Automotive & $\%$ & 0 & 6 & 2 & 13 & 0 & 5 \\
Others & $\%$ & 1 & 18 & 16 & 35 & 14 & 20 \\
\hline Total & $\%$ & $\mathbf{1 0 0}$ & $\mathbf{1 0 0}$ & $\mathbf{1 0 0}$ & $\mathbf{1 0 0}$ & $\mathbf{1 0 0}$ & $\mathbf{1 0 0}$ \\
\hline MS $(Q)_{i}$ value & & & & & & & \\
$Q=$ High & - & 1 & 1 & 1 & 1 & 1 & 1 \\
$Q=$ Medium & - & 0.43 & 0.73 & 0.46 & 0.82 & 0.85 & 0.66 \\
$Q=$ Low & - & 0.43 & 0.70 & 0.42 & 0.76 & 0.74 & 0.60 \\
\hline
\end{tabular}

* Average of market shares on the other polymer markets weighted according to polymer abundance in Europe.

\section{$<$ heading level $1>$ Results and discussion}

\section{$<$ heading level $2>$ Resource recovery efficiencies}

Four types of resource recovery efficiency were calculated and illustrated in figure 2 for all scenarios, including: 1) all MRF outputs with impurities (A), 2) all MRF outputs without impurities ( $\triangle$ ), 3) monopolymer outputs (PET, HDPE, PP, and PS) with impurities (ם) and 4) mono-polymer outputs without impurities ( $\square$ ). As the reprocessing efficiency was not included in the calculation of the resource recovery efficiencies, these represents the best case scenario, where loss during reprocessing is close 
to 0 . The scenarios on the $\mathrm{x}$-axis were ordered first according to the sorting scheme (A-F), and then according to increasing source-separation efficiencies, followed by increasing MRF performance, reflecting that the combination of source-separation and MRF efficiencies increases within each sorting scheme. A detailed scenario list is given in SI, table S4. The achieved range of resource recovery efficiencies within each sorting scheme reflected the potential performance of that specific sorting scheme based on data available in the literature.

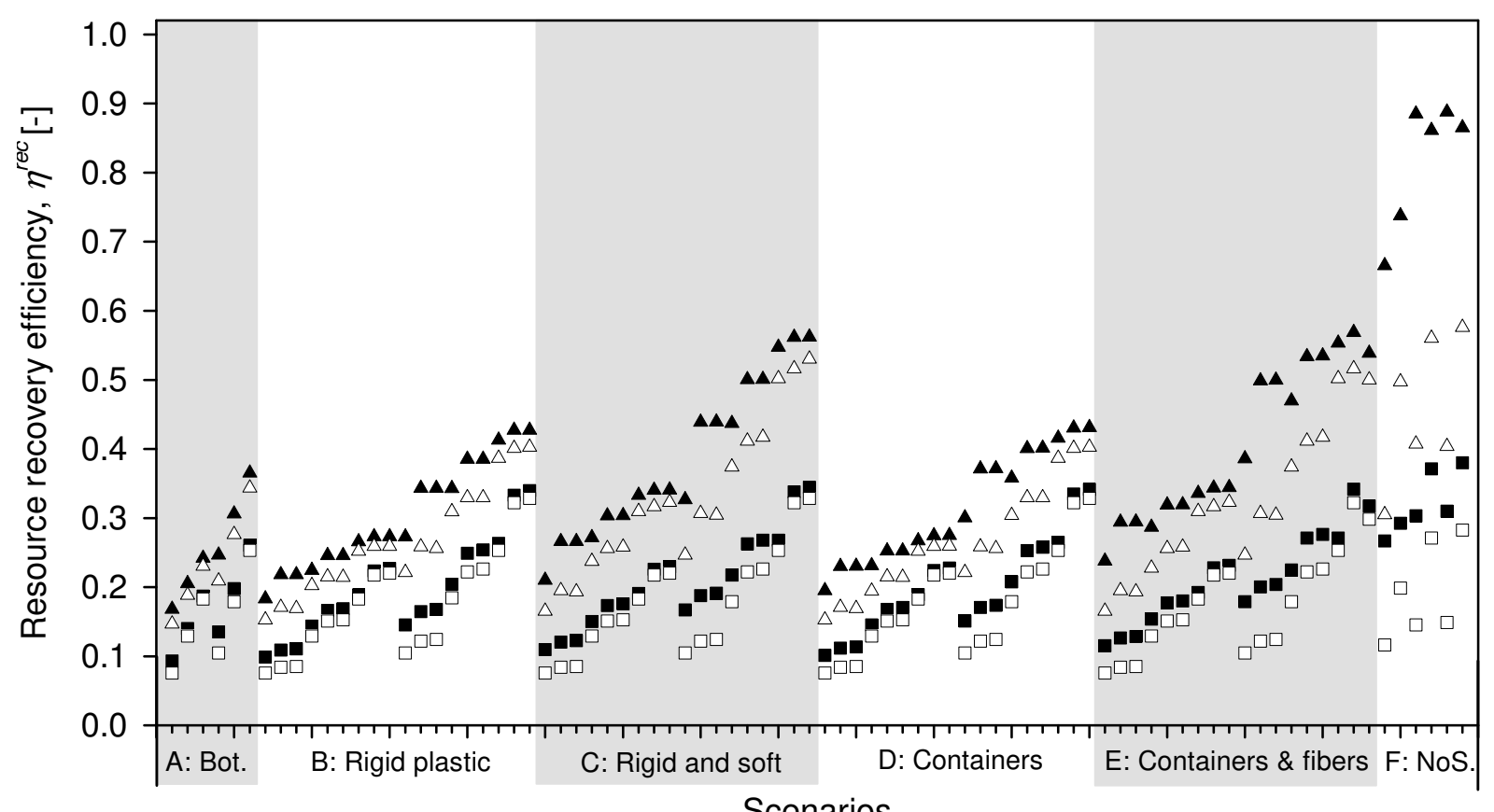

\section{Scenarios}

Figure 2 Resource recovery efficiency, $\eta^{\text {rec }}$, for all scenarios, calculated using eq. (1). Resource recovery efficiencies are depicted both with and without impurities as well as including all MRF outputs or only mono-polymer outputs (PET, HDPE, PP, and PS). The scenarios are presented according to sorting scheme (Bot.: Bottles, NoS: No source-separation). Within each sorting scheme, the scenarios are ordered according to increasing source-separation and MRF efficiencies. The specific order is given in the SI, table S4. 

found between 0.17 and 1.0, which means that between $17 \%$ and $100 \%$ of the plastic mass generated in the household were recovered at the MRF and prepared for recycling, when including impurities in the calculations. This is a large variation, depending on several parameters. In addition to the sourceseparation and MRF efficiencies themselves, the overall sorting scheme was important, as scenarios only targeting bottles and rigid plastic (A, B, D) had lower recovery efficiencies (i.e. 0.17-0.44) than scenarios additionally targeting plastic film (C, E), (i.e. 0.22-0.58), which again were lower than scenarios where all plastic was collected and routed to the MRF with residual waste (F), (i.e. 0.78-1.0).

Consequently, reaching recovery efficiencies above $50 \%$ for plastic from HHW requires efficient source-separation of rigid and soft plastic fractions, while very high recovery efficiencies only appear possible through "dirty MRF" solutions. However, excluding impurities from the resource recovery efficiencies $(\triangle)$ resulted in considerable decreases to $0.15-0.58$ across all sorting schemes. This indicates that a substantial part of the recovered plastic from "dirty MRF" systems represented impurities, and that impurities carried along with the target fractions may be important in discussions about recycling rates (GBB, 2015; Haupt et al., 2017). efficiencies with $(\boldsymbol{\Delta})$ and without $(\triangle)$ impurities can be observed. As the level of non-plastic impurities increases with increasing numbers of target fractions, the share of non-plastic impurities for scenarios with sorting scheme $\mathrm{F}$ was between $38 \%$ and $61 \%$, while this share was only between $5 \%$ and $17 \%$ for scenarios with sorting scheme A. Consequently, the scenarios with no source-separation (F) only recovered slightly more plastic than scenarios with separation schemes including both rigid and soft plastic (i.e. C, E). These scenarios recovered considerably more plastic than scenarios only targeting rigid plastic (i.e. A, B, D). Within each sorting scheme, in particular A-E, the wide range in resource recovery efficiencies illustrates that a well-performing source-separation and MRF system is crucial for 411 achieving competitive performance in comparison with alternatives. 
across all sorting schemes (A-F). As such, the recovery of single polymer streams depended mainly on the source-separation and MRF efficiencies. Mono-polymer outputs represent a primary target for recovered plastic, as these fractions may potentially be recycled into high or medium quality material, while plastic fractions with unknown polymer compositions (especially mixed plastic) may be recycled only into low quality material (discussed in details in the next section) (Dvorak et al., 2009; Luijsterburg and Goossens, 2014). The higher overall resource recovery efficiencies for scenarios including film (C, E), or scenarios with no source-separation $(F)$, therefore reflected the recovery of mixed plastic fractions (film and mixed plastic), which can be recycled only as lower quality material.

The results demonstrate that in order to achieve high overall recovery, source-separation efficiencies for target materials of around 60-90\%, and a well-performing MRF with recovery efficiencies of target material to the intended outputs of around $75-95 \%$ are paramount.

\section{$<$ heading level 2> Quality of recovered MRF outputs}

Figure 3a illustrates the shares of recovered high, medium, or low quality plastic, as well as the shares of plastic too contaminated to be recycled at all. The upper limit of the bars in figure $3 \mathrm{a}$ is identical to the resource recovery efficiency including all MRF outputs with impurities $(\mathbf{\Delta})$ in figure 2.

In all scenarios but four, at least one of the MRF outputs was found unsuitable for recycling, due to the level of impurities, and at best $55 \%$ of the plastic generated in the household was found suitable for recycling after mechanical sorting. For most scenarios this was caused by the mixed plastic fraction which was too contaminated to be recycled. For many scenarios more than one output was found unsuitable for recycling and as a result the potentially recycled amounts were much lower than what the resource recovery efficiencies in figure 2 suggested. This was especially pronounced for scenarios with sorting scheme F with no source-separation. Although they recovered the largest share

438 of plastic, only a small fraction - if any - was suitable for recycling. In 26 scenarios, all the recovered

439 MRF outputs were found unsuitable for recycling, due to poor MRF performance resulting in large 440 amounts of missorted items and non-targeted polymers in the recovered MRF outputs. This reflects that the MRF performance is crucial for the overall level of recycling. 
With respect to the quality of the recovered plastic suitable for recycling, again, MRF performance appear critical for the results. For scenarios with low-performing MRFs, the recovered plastic may at best be low quality. On the other hand, scenarios recovering plastic with a potential for recycling into high quality all involve high performing MRFs. However, high recovery efficiencies at the MRF are not the only factor necessary to ensure recycling of high-quality recycled plastic from households - this moreover requires that the reprocessing facility passes a challenge test, demonstrating a sufficiently effective decontamination process (EC, 2008). Based on current European recycling practices, only streams of PET bottles including maximum 5\% non-food products collected separately from the remaining plastic HHW are, to our knowledge, approved for recycling into high-quality foodgrade products (EFSA, 2018). the largest differences are found within the sorting schemes rather than between them. On this basis, however, the trend remains that scenarios with the largest shares of recovered plastic suitable for recycling are those with a sorting scheme targeting soft plastic (i.e. C, E). The overall best-performing scenarios, with respect to both the quantity and the quality of outputs, are hence the ones with sorting schemes $\mathrm{C}$ and $\mathrm{E}$, with high-performing MRFs producing all possible MRF outputs (including film recovery). Yet, none of these scenarios included a mixed plastic fraction suitable for recycling. Detailed data showing the quality of all recovered outputs in all scenarios can be found in SI, tables S15 and S16. 

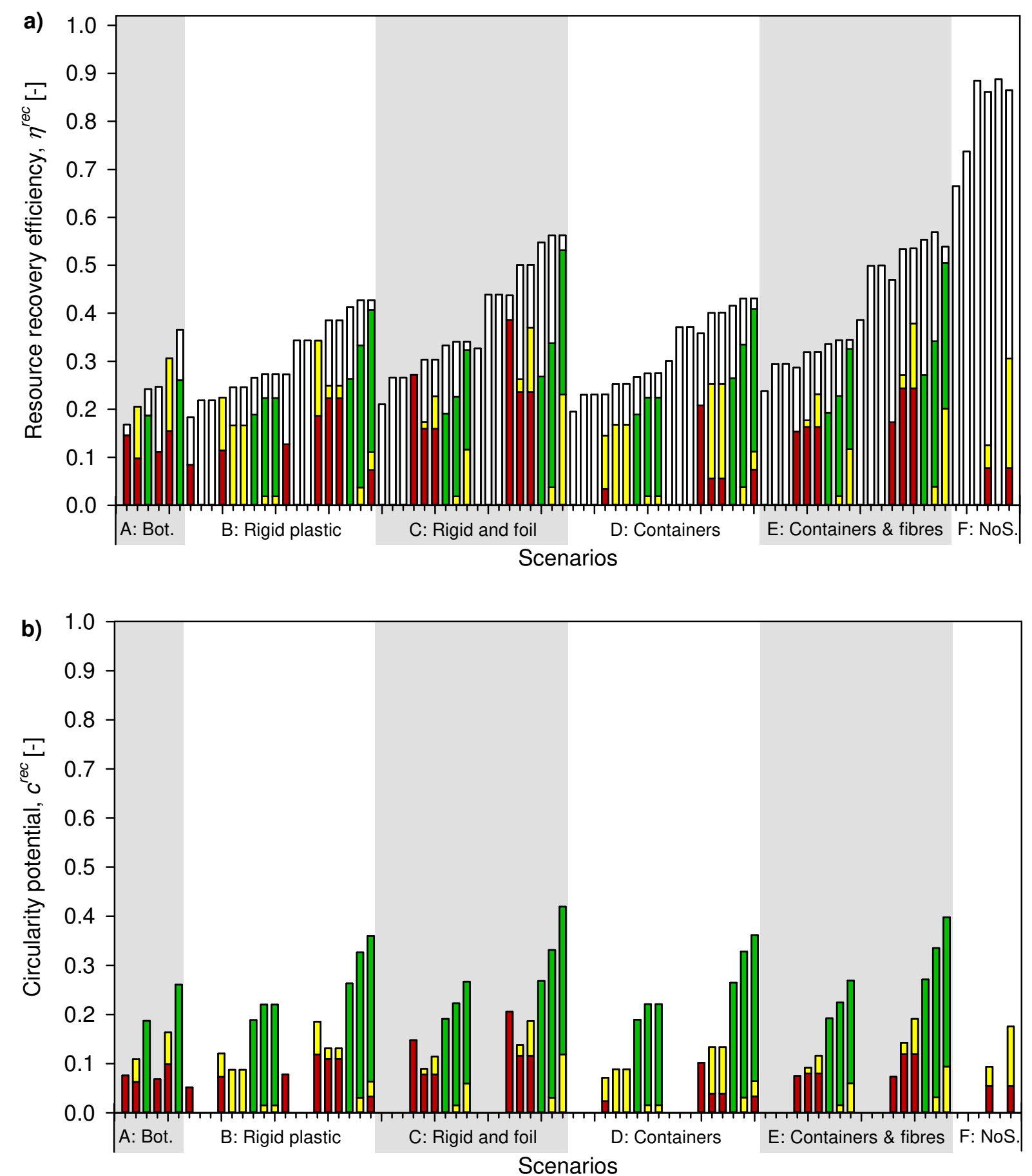

$\square$ Not recyclable $\square$ High quality $\quad \square$ Medium quality $\quad \square$ Low quality

462 Figure 3 a) Resource recovery efficiencies, $\eta^{\text {rec }}$, [-] for all scenarios, indicating the quality of the 463 recovered outputs. The top of the bar corresponds to $\eta^{r e c}, \boldsymbol{\Lambda}$, in figure 2. Excluding outputs not suitable 464 for recycling $(\square)$, the recyclable fraction is obtained $(\square++\square)$. b) circularity potential, $c^{\text {rec }}$, for all 465 scenarios [-], indicating the quality of the contributing outputs. The scenarios are presented according to sorting scheme (Bot.: Bottles, NoS: No source-separation). Within each sorting scheme, the scenarios 
are ordered according to increasing source-separation and MRF efficiency. The specific order is given in the SI, table S4.

\section{$<$ heading level $2>$ Potential circularity of scenarios}

From the results presented in figure $3 \mathrm{a}$, the circularity potential for all scenarios were determined based on eq. (2) and presented in figure 3b. The scenarios including the recovery of PET and HDPE outputs to high quality are those with the highest circularity potential, as the high quality PET and HDPE outputs have the potential to substitute virgin plastic in all possible applications on the PET and HDPE markets, whereas medium- and low-quality PET and HDPE may only substitute virgin plastic in parts of these markets. As more than half of the PET market relies on high-quality PET for food packaging production, the reduction in circularity potential when going from high to medium or low-quality is especially prominent for PET.

The contribution made by film to the overall circularity potential is considerably lower than suggested by the resource recovery efficiencies alone: Even in the best-performing scenarios it was only possible to recycle the film fraction into medium-quality with a potential to substitute virgin plastic in non-food contact applications. As food contact applications represent more than half of the film market, this limitation in recycling is critical. Nevertheless, scenarios targeting film and involving high sourceseparation and MRF efficiencies offer the best overall circularity potential $\left(c^{\text {rec }}=0.42\right)$, as the amount of recyclable waste recovered was high (due to film) and a considerable share of the recovered waste had a potential to be recycled into high-quality (due to PET and HDPE).

While scenarios with no source-separation (sorting scheme F) had the highest overall resource recovery efficiencies (figure 2 and $3 a$ ), the low circularity potentials clearly demonstrate the challenges associated with this recovery approach (figure $3 b$ ): as the generated plastic outputs only allow recycling into medium or low-quality the ability to close plastic loops is limited. Although these scenarios recovered similar recyclable plastic amounts as the better performing scenarios with a different sorting schemes (A-E), the quality of the recovered materials was lower, thereby decreasing the circularity potential considerably. Consequently, although recycling pathways allowing the mechanical separation of plastic from residual waste in large amounts exist, the quality of the recovered plastic limits recycling, 
and only partial potential to substitute virgin plastic within the individual polymer markets can be expected, thereby only closing the individual polymer loops partially, if at all.

\section{$<$ heading level 2> Implications of circularity and potential substitution}

The value of $c^{r e c}$ represents the circularity potential of a recovery or recycling system when the individual polymer markets are in steady-state. Although debatable whether this is realistic or not, steady-state is an integral assumption in the vision of closed material loops, and as such a condition that should be reflected when evaluating the circularity potential. However, at a European scale steady-state polymer markets are far from reality. Fellner at al. (2017) estimated that - theoretically - only around half of the European plastic demand can be covered by recycling of plastic waste generated in Europe (assuming no plastic losses in the recycling system). As illustrated by figure 3a considerable losses are related to current recycling of plastic from HHW, and thus most likely significantly less than half of the demand can be covered by recycled plastic. Consequently, low quality recycled plastic may well be absorbed by the current polymer market and substitute virgin plastic, even if the market for low quality materials only represents $40-50 \%$ of the total market (in case of PET and LDPE, see table 5). Recycling systems converting plastic items of high-quality into low-quality plastic may therefore be sufficient in the current situation and as the first phase of a transition towards a circular economy. However, only implementing systems with low or medium quality outputs will not offer a long-term solution towards circularity and closed material loops, as substitution of virgin plastic in significant shares of the polymer markets will not be possible.

The circularity potential, as presented in figure $3 \mathrm{~b}$, offers an approach to quantify the "circularity" of recovery systems involving waste materials such as plastic from HHW. However, assessing large-scale recovery or recycling systems, e.g. at European level assuming steady-state polymer markets in a long-term perspective, the substitution potential of the recovery systems should approach the circularity potential. Consequently, based on $1 \mathrm{~kg}$ of plastic waste generated in households

520 figure $3 \mathrm{~b}$ shows that at best $0.42 \mathrm{~kg}$ virgin material may be substituted (excluding losses during reprocessing). While complete circularity (albeit a theoretical concept) would require a 1:1 substitution 
managing plastic from HHW is far from effective in a long-term transitioning towards complete closed plastic loops.

Based on the results, three fundamental pathways are possible to move towards closed material loops: 1) sorting and recovery technologies has to be significantly improved, 2) plastic flows including high quality items have to be managed separately from the remaining waste including lower qualities to increase likelihood of maintaining quality, and 3) the design of plastic products has to be significantly improved to allow better separation and recovery. The amount of impurities entering the recycling systems should be minimized as much as possible to minimize both physical and quality losses throughout recycling. Improving source-separation and technology efficiencies as well as designing new products for recycling are essential in this context. It is necessary to view plastic not as one bulk material but acknowledge the presence of a wide range of qualities and polymers. To further improve the system circularity an increased focus on individual polymers and product categories with potentials for high quality recycling (e.g. similar to existing recycling of PET bottles) may be needed.

\section{$<$ heading level 1 $>$ Conclusion}

A novel approach for quantifying the circularity potential of material recovery and recycling systems is presented and applied on scenarios involving recovery of plastic from household waste. The circularity potential was defined as a function of the resource recovery efficiency and the ability of individual recovered fractions to fulfill quality demands in a steady-state market representing a closed material loop situation. Higher resource recovery efficiencies and inclusion of more target fractions in the separation scheme, especially film, offered higher resource recovery efficiencies overall, with the MRF efficiency as the single most important parameter. For many scenarios, several of the recovered plastic fractions were unsuitable for recycling due to detrimental levels of impurities. Consequently, in the best performing scenarios only $55 \%$ of the generated plastic waste was recovered in a MRF output suitable for recycling. Particularly mixed plastic fractions containing several polymers were found unsuitable for recycling. The circularity potential indicated that the best-performing plastic recovery system (sorting schemes including rigid and soft plastic, the highest number of polymer target fractions, and high source-separation and MRF efficiencies) had potential to close $42 \%$ of the material loop. This 
suggests that with current technology, Europe is far from able to close the plastic loop (requiring a theoretical circularity potential of 1). To improve the situation, the presence of impurities in the recovered fractions should be reduced and more emphasis should be placed on closing the loops for high quality plastic rather than plastic in general.

\section{[Heading 1] Acknowledgement}

Funding from The Danish Environmental Protection Agency and the IRMAR project (Grant no. 11$116775)$ is gratefully acknowledged.

\section{[Heading 1] References}

Ballesteros-Gómez, A., Brandsma, S.H., de Boer, J., Leonards, P.E.G. 2014. Direct probe atmospheric pressure photoionization / atmospheric pressure chemical ionization high-resolution mass spectrometry for fast screening of flame retardants and plasticizers in products and waste. Analytical and Bioanalytical Chemistry, 406(11): 2503-2512

Braungart, M., McDonough, W., 2002. Cradle to Cradle: Remaking the Way We Make Things. New York. North Point Press.

Cappadona, B., 2015. Residential Recycling Top Contaminants [online lecture]. US Environmental Protection Agency, Sustainable Materials Management Webinar, December 172015 [5:0025:22]. https://www.youtube.com/watch?v=-rS2F9r_lGE\&feature=youtu.be [6 September 2018].

Cimpan, C., Maul, A., Jansen, M., Thomas, P., Henrik, W. 2015. Central sorting and recovery of MSW recyclable materials: A review of technological state-of-the-art, cases, practice and implications for materials recycling. Journal of Environmental Management 156(June): 181199

Dahlbo, H., Poliakova, V., Mylläri, V., Sahimaa, O., Anderson, R. 2017. Recycling potential of postconsumer plastic packaging waste in Finland. Waste Management 71: 52-61 
Dvorak, R., Evans, R., Kosior, E., 2009. Commercial scale mixed plastics recycling. UK: WRAP.

EC (European Commission), 2008. EC/282/2008 on recycled plastic materials and articles intended to come into contact with foods of 27 March 2008. Official Journal of the European Union. L86, $9-18$.

EC (European Commission), 2015. Proposal for a DIRECTIVE OF THE EUROPEAN PARLIAMENT AND OF THE COUNCIL amending Directive 2008/98/EC on waste. Brussels. EC (European Commission), 2018a. DIRECTIVE (EU) 2018/851 OF THE EUROPEAN PARLIAMENT AND OF THE COUNCIL amending Directive 2008/98/EC on waste of 30 May 2018. Official Journal of the European Union. L150, 109-139.

EC (European Commission), 2018b. DIRECTIVE (EU) 2018/852 OF THE EUROPEAN PARLIAMENT AND OF THE COUNCIL amending Directive 94/62/EC on packaging and packaging waste of 30 May 2018. Official Journal of the European Union. L150, 141-154.

EC (European Commission), 2018c. Turning today's challenges into opportunities - a European strategy for plastics in a circular economy. Factsheets on A European Strategy for Plastics in a Circular Economy. Brussels: European Commission.

Edjabou, M.E., Jensen, M.B., Götze, R., Pivnenko, K., Petersen, C., Scheutz, C., Astrup, T.F., 2015. Municipal solid waste composition: Sampling methodology, statistical analyses, and case study evaluation. Waste Management, 36: 12-23.

EFSA (European Food Safety Authority), 2018. Register of questions [online register]. Unit filter: Food Ingredients and Packaging, keyword: Recycling. http://registerofquestions.efsa.europa.eu/raw-war/ListOfQuestionsNoLogin?1 [6 September 2018]

EMF (Ellen MacArthur Foundation), 2013. Towards the circular economy - Economic and business rationale for an accelerated transition [online report]. Ellen MacArthur Foundation. https://www.ellenmacarthurfoundation.org/assets/downloads/publications/Ellen-MacArthurFoundation-Towards-the-Circular-Economy-vol.1.pdf [6 September 2018]

Ernst, T., Popp, R., van Eldik, R. 2000. Quantification of heavy metals for the recycling of waste plastics from electrotechnical applications. Talanta 53(2): 347-357 
Fellner, J., Lederer, J., Scharff, C., Laner, D. 2017. Present Potentials and Limitations of a Circular Economy with Respect to Primary Raw Material Demand. Journal of industrial ecology 21(3): 494-496

Feil, A., Pretz, T., Jansen, M., van Velzen, E.U.T., 2016. Separate collection of plastic waste, better than technical sorting from municipal solid waste? Waste Management \& Research, 35(2): 172180.

GBB (Gershman, Brickner \& Bratton, Inc.), 2015. The Evolution of Mixed Waste Processing Facilities 1970-Today. Fairfax, Virginia: American Chemistry Council. https://plastics.americanchemistry.com/Education-Resources/Publications/The-Evolution-ofMixed-Waste-Processing-Facilities.pdf [6 September 2018].

Götze, R., Astrup, T. 2013. Characterisation of Plastic Packaging Waste for Recycling: Problems Related To Current Approaches. Paper presented at the Fourteenth International Waste Management and Landfill Symposium, 30 September - 4 October 2013, Sardinia

Gu, F., Guo, J., Zhang, W., Summers, P.A., Hall, P. 2017. From waste plastics to industrial raw materials: A life cycle assessment of mechanical plastic recycling practice based on a real-world case study. Science of the total environment 601: 1192-1207

Haupt, M., Vadenbo, C., Hellweg, S., 2017. Do we have the right performance indicators for the circular economy? - Insight into the Swiss waste management system. Journal of Industrial Ecology 21(3): 615-627.

Heinzel, J.V., Larsen, C.S., Tønning, K., Malmgren-Hansen, B., Nilsson, N.H., 2015. Mekanisk sortering af plastaffald fra husholdninger (Mechanical sorting of plastic waste from households) [report]. Copenhagen: Danish Environmental Protection Agency.

Huber, M., Franz, R. 1997. Identification of Migratable Substances in Recycled High Density Polyethylene Collected from Household Waste. Journal of High Resolution Chromatography. http://doi.org/10.1002/jhrc.1240200806

Huysman, S., De Schaepmeester, J., Ragaert, K., Dewulf, J., De Meester, S., 2017. Performance indicators for a circular economy: A case study on post-industrial plastic waste. Resources, conservation and recycling. http://doi.org/10.1016/j.resconrec.2017.01.013 
Luijsterburg, B., Goossens, H. 2014. Assessment of plastic packaging waste: Material origin, methods and properties. Resource, Conservation and Recycling. http://doi.org/10.1016/j.resconrec.2013.10.010

Mengarelli, M., Neugebauer, S., Finkbeiner, M., Germani, M., Buttol, P., Reale, F. 2017. End-of-life modelling in life cycle assessment—material or product-centred perspective? International journal of life cycle assessment. http://doi.org/10.1007/s11367-016-1237-z

Papineschi, J., Tilbrook, E., Emery, L. 2016. Review of the Welsh government collections blueprint [report]. Eunomia Research \& Consulting Ltd.

Petersen, C., Mayland, C., 2015. Genanvendeligt affald i indsamlet dagrenovation (Recyclable waste in collected residual household waste) [report]. Danish Envi-ronmental Protection Agency.

Petersen, C., Kaysen, O., Edjabou, V., Manokaren, S., Tønning, K., Hansen, T., 2012. Kortlægning af dagrenovation i enfamilieboliger [Characterization of re-sidual waste in single family houses]. Danish Environmental Protection Agen-cy.

Petersen, C., Kaysen, O., Manokaren, S., Tønning, K., Hansen, T., 2014. Kortlægning af dagrenovation i Danmark [Characterization of residual waste in Denmark]. Danish Environ-mental Protection Agency.

Petersen, C., Mayland, C., Manokaran, S. 2015. Plast fra restaffald - analyse af restaffald med fokus på plast (Plastic from residual waste - analysis of the residual waste with focus on plastic). EcoNet and ARC.

Pivnenko, K., Eriksen, M.K., Martín-Fernández, J.A., Eriksen, E., Astrup, T.F. 2016. Recycling of plastic waste: Presence of phthalates in plastics from households and industry. Waste management 54: 44-52

PlasticsEurope, EPRO (European Association of Plastic Recycling and Recovery Organisations) 2016. Plastics - the Facts 2016. An analysis of European plastics production, demand and waste data https://www.plasticseurope.org/application/files/4315/1310/4805/plastic-the-fact-2016.pdf [6 September 2018]

Pressley, P.N., Levis, J.W., Damgaard, A., Barlaz, M.A., DeCarolis, J.F. 2015. Analysis of material recovery facilities for use in life-cycle assessment. Waste Management 35: 307-317 
Ragaert, K., Delva, L., Geem, K. 2017. Mechanical and chemical recycling of solid plastic waste. Waste Management 69: 24-58

Riber, C., Petersen, C., Christensen, T.H. 2009. Chemical composition of material fractions in Danish household waste. Waste management 29(4): 1251-1257

Rigamonti, L., Grosso, M., Sunseri, M. C. 2009. Influence of assumptions about selection and recycling efficiencies on the LCA of integrated waste management systems. International Journal of life cycle assessment 14(4): 411-419

Rigamonti L., Grosso, M., Møller, J., Martinez Sanchez, V., Magnani, S., Christensen T. H., 2014. Environmental evaluation of plastic waste management scenarios. Resources, Conservation and Recycling 85: 42-53

Rigamonti, L., Niero, M., Haupt, M., Grosso, M., Judl, J. 2018. What happens during recycling processes and what are the implications on the quality of secondary materials? Food for thought for waste-management-oriented LCA. Waste Management Article in press.

Seyring, N., Dollhofer, M., Weißenbacher, J., Herczeg, M., McKinnon, D., Bakas, I., 2015. Assessment of separate collection schemes in the 28 capitals of the EU. Brussels: European Commission.

Shen, L., Worrell, E., Patel, M. 2010. Open-loop recycling: A LCA case study of PET bottle-to-fibre recycling. Resources, Conservation and Recycling 55(1): 34-52

Vadenbo, C., Hellweg, S., Astrup, T. F. 2016. Let's be clear(er) about substitution - a reporting framework to account for product displacement in Life Cycle Assessment. Journal of Industrial Ecology 21(5): 1078-1089

van der Harst, E., Potting, J., Kroeze, C., 2016. Comparison of different methods to include recycling in LCAs of aluminium cans and disposable polystyrene cups. Waste management 48: 565-583

Vilaplana, F., Karlsson, S., 2008. Quality concepts for the improved use of recycled polymeric materials: A review. Macromolecular Materials and Engineering 293(4): 274-297

Villanueva, A., Eder, P., 2014. End-of-Waste Criteria for waste plastic for conversion. Luxembourg: Publications Office of the European Union. 
689 Whitt, M., Vorst, K., Brown, W., Baker, S., Gorman, L. 2012. Survey of heavy metal contamination in recycled polyethylene terephthalate used for food packaging. Journal of Plastic Film and

691 Sheeting 29(2): 163-173

692

693 


\section{:/I SUPPORTING INFORMATION FOR:}

Eriksen, M. K., Damgaard, A. Boldrin, A., Astrup, T. F. 2018. Quality assessment and circularity potential of recovery systems for household plastic waste. Journal of Industrial Ecology.

\section{Summary}

This supporting information includes four sections: Section S1: Detailed description of scenarios, Section S2: Quality assessment, Section S3: Identification of European market shares and Section S4: Results, as presented below in the table of content.

\section{Table of Content}

Section S1: Detailed description of scenarios ........................................................ 2

S1.1: Overall sorting scheme ............................................................... 2

S1.2: Source-separation and collection................................................... 4

S1.3: Material recovery facility (MRF) ............................................... 5

S1.3.1: Recovery efficiencies ..................................................... 6

S1.3.2: Estimation of efficiencies for non-target materials ................. 11

S1.4: Outputs.............................................................................. 12

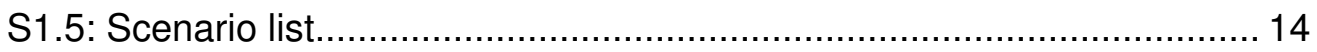

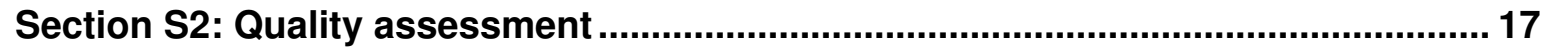

S2.1: Definition of application groups ............................................. 17

S2.2: Quality criteria and assessment .............................................. 18

Section S3: Identification of European market shares ........................................... 26

S3.1: Food and non-food packaging .................................................... 26

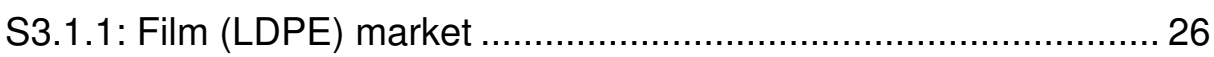

S3.1.2: PET, HDPE, PP, PS market ............................................. 26

S3.2: Toys, Pharmaceuticals and Others ............................................. 29

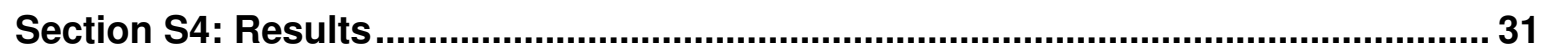

S4.1: Resource recovery efficiencies - MRF outputs and quality ...............31

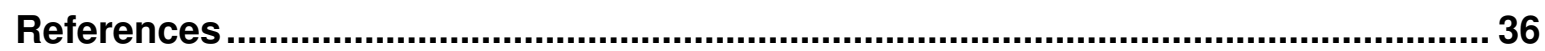




\section{Section S1: Detailed description of scenarios}

The scenarios consists of four scenario steps with different choices that vary from scenario to scenarios. An overview is given in Figure 1 in the main paper or below. In the following the different scenario steps and the reasoning for the different choices within the scenario steps are described in details. Finally, an exhaustive list of all the scenarios included in and excluded from the study is given in Section S1.5.

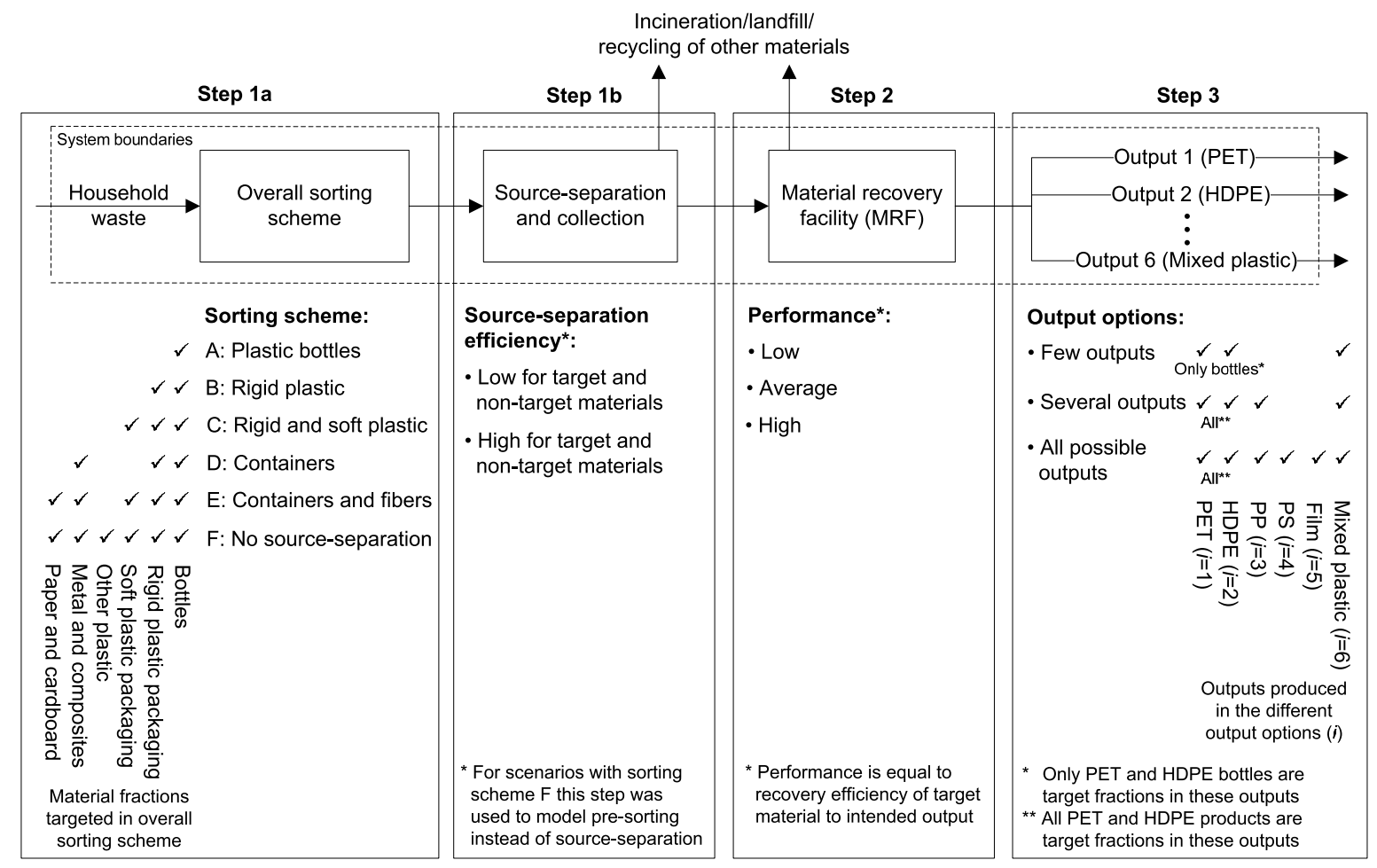

Figure 1 Possible scenario configurations. The different choices for each step are stated below the step. Material fractions included in the overall sorting scheme are indicated (step 1a), as well as targeted fractions in the different output choices (step 3). Containers refer to plastic, metal, and composite containers. $i$ refers to eq. (1) and (2) in the main paper.

\section{S1.1: Overall sorting scheme}

Six overall sorting schemes were identified (A-F) as typical sorting schemes in Europe, which are explained below.

Sorting scheme $A$ was the one including the smallest number of items: plastic bottles. Plastic bottles are easily recognisable from the rest of the household waste and consist of only a few polymer types (mainly PET and HDPE, see Table 1 in the main paper) and thus represents a separation scheme easy for the citizens to use.

Sorting scheme $B$ included separation of all rigid plastic packaging, i.e. plastic bottles and other rigid plastic packaging items, such as trays, containers, etc. This type of 
separation scheme also includes rigid plastic items of PP, PS and other polymer types (see Table 1 in the main paper) and is implemented several places in Europe.

\section{Sorting scheme $C$ included separation of foil and rigid plastic packaging and does} therefore also include LDPE items, as soft plastic in the HHW is most often made from LDPE (see Table 1 in the main paper). Several municipalities in Denmark and the Netherlands are examples of where this kind of sorting scheme is currently in use (Luijsterburg and Goossens, 2014).

Sorting scheme $D$ was a comingled system including separation of containers of both rigid plastic, metal and composite material. This kind of system is often call a dualstream commingled system and has been used for several decades in Germany and is currently also in use in $10 \%$ of the municipalities in the United Kingdom (Feil et al., 2016; Cimpan et al., 2015). Due to the addition of non-plastic material this sorting scheme does, besides plastic items of PET, HDPE, PP, PS and other polymers, also include metal and composite items.

Sorting scheme $E$ represented a fully commingled system where all dry recyclables, including both containers (plastic, metal, composites and sometimes glass), plastic foil and fibres (paper and cardboard) are separated into one bin in the household. This is often called a single-stream commingled system and is a separation scheme gaining popularity in several countries in Europe. For example, $98 \%$ of the private households in Ireland are currently using this kind of separation system (Cimpan et al., 2015).

Sorting scheme $F$ was fundamentally different from the others since it does not require the citizens to separate any recyclables in the household. Here all the residual waste, including all the waste plastic items generated in the households, is collected and send to a specialised separation facility, where the plastic is mechanically presorted (Feil et al., 2016), increasing the concentration of the plastic in the waste stream subsequently send to actual plastic sorting at a MRF. This is also known as mechanical biological treatment (MBT) or dirty MRF. This system is used several places in Europe, e.g. $22 \%$ of the municipalities in the Netherlands separate their plastic waste from the residual waste using mechanical sorting (Bing et al., 2014) and it is gaining popularity, since it does not require the citizens to do any separation and all recyclables are collected at once, having the potential of achieving high plastic recovery rates. 


\section{S1.2: Source-separation and collection}

Scenario step 1B was related to the source separation and thereby the collection efficiency. Only few recent studies provide information related to collection efficiencies for plastic fractions in household waste, including Petersen and Manokaran (2013), van Velzen et al. (2013), Rigamonti et al. (2014), and Cimpan et al. (2015). However, the majority of these studies only report measured collection efficiencies for a specific area. As collection efficiencies strongly depend on local conditions they are expected to vary greatly from one area to another. Consequently, a high and a low sorting efficiency for each target material fraction were determined based on the aforementioned studies, to take the variability due to differences in local conditions, willingness from the citizens to participate, maturation of the sorting scheme, etc. into account. All collection efficiencies used when defining the second scenario step are presented in Table 3 in the main paper.

For scenarios with sorting scheme $F$, step $1 \mathrm{~b}$ was used to model a mechanical pre-sorting step instead of source-separation in the household. The purpose of the pre-sorting was to upconcentrate the plastic in a "primary plastic flow" send to actual plastic recycling. The share of non-plastic items in the "primary plastic flow" after pre-sorting has been reported previously at $28-49 \%$ (Feil et al., 2016). Some of these non-plastic items represent targeted non-plastic materials for recycling in a "dirty MRF" context rather than missortings (e.g. paper is a target fraction in a "dirty MRF" and the sorting is to a certain degree designed to catch paper from the non-paper streams and redirect it to the paper output). Hence, to reflect this, the share of non-plastic items entering the MRF as missortings was assumed to be $28 \%$ for sorting scheme $F$.

Besides the collection efficiencies of the target materials the table furthermore presents collection efficiencies of non-plastic items, i.e. material fractions that, according to the sorting scheme, were not supposed to be included in the source separation. The collection efficiency of non-plastic items were assumed to range from $0.001 \%$ to $0.02 \%$ and be low in the scenarios where the collection efficiencies of the target materials were low and vice versa.

As no quantitative data currently exist in relation to the collection efficiencies of nontarget fractions, these collection efficienices were estimated based on reported impurities in the source separated stream collected for sorting. It was assumed that the collection efficiencies for non-targeted items varied depending on the sorting scheme of the scenario, as several sources claimed that the percentage of contaminants received at MRFs is smaller for sorting scheme only including plastic items (A: plastic bottles, B: rigid plastic and C: rigid and soft plastic) compared to sorting schemes including both plastic and other materials (D: containers and E: containers and fibres) (Cappadona, 2015; Moreau, 2015). The 
contamination ranges was assumed to vary from $2 \%$ in the systems solely targeting bottles (A: plastic bottles) to $19 \%$ in the scenarios where all dry recyclables were collected together (E: Containers and fibres), based on studies reporting $1.5 \%$ contamination in a source separated plastic bottle stream from a recycling centre (Heinzel et al., 2015) and up to 18.9\% impurities in the input to single stream MRF (corresponding to sorting scheme E) (Enviros, 2009). However, since data related to the level of impurities in collected source separated fractions are very scarce in literature, these assumption are quite rough, underlining the need for more data related to the impurities.

\section{S1.3: Material recovery facility (MRF)}

Scenario step 2 was related to the technical performance of the MRF. A MRF can be designed in numerous different ways depending on the number of sorting steps, the type(s) of technology, the efficiency of the technologies, the efficiency of the staff in relation to potential manual sorting, etc. (Enviros, 2009) and consequently the performance and thereby the recovery efficiencies are expected to vary considerably depending on the design of the specific MRF. Moreover, the overall performance and the performance of the individual equipment is assumed to depend on the composition of the incoming waste. For example, plastic foil can decrease the efficiency and ultimately damage the sorting equipment, if the MRF is not design to separate foils (Cappadona, 2015) and contamination of the surface of plastic items can decrease the efficiency of NIR sorting equipment (Petersen and Mayland, 2015).

However, information related to the overall performance of MRFs are scarce in literature and only very limited information exists outside academia, often related to the same kinds of MRF, originating from the same interest organisations. For example several studies, initiated by WRAP, are related to UK MRFs solely receiving commingled recyclables (Shonfield, 2008; Enviros, 2009; LRS consultancy, 2015). It was therefore not possible, based on the information available, to define MRF performances that were dependent on the sorting scheme or the incoming waste composition.

Consequently, generic recovery efficiencies for low, average and high performing MRFs were defined and used in all scenarios regardless of the sorting scheme. The recovery efficiencies were modelled as transfer coefficients, of the different material fractions and polymer types to the different outputs produced by the MRF. The high and low efficiencies of the target materials were defined inspired by the most extreme values found in literature (Goodman, 2006; Shonfield, 2008; Dvorak et al., 2009; Enviros, 2009; Spendelow, 2011; Jansen et al., 2012; van Velzen et al., 2013; LRS consultancy, 2015; Cimpan et al., 2015; 
RRS, 2015) whereas the average efficiency was calculated as an average of the high and the low efficiency.

As most of the studies only reported recovery efficiencies of target materials (e.g. PET bottles to PET output), it was necessary to estimate the recovery efficiencies of non-target materials (e.g. PE or non-plastic items to a PET output). Recovery efficiencies of all target and non-target materials are presented in Table S1-S3 and it is explained in details how the non-target recovery efficiencies have been estimated in section S1.3.2.

\section{S1.3.1: Recovery efficiencies}

In Table S1-S3 the recovery efficiencies [-] for low, medium and high performing MRFs are presented based on MRF outputs. Recovery efficiencies are given for all parts of the waste composition (divided based on both polymer type and product category) to a specific output. Adding the recovery efficiency of a specific fraction, for a specific performance for all possible MRF outputs, gives the overall recovery efficiency of that MRF. The remaining fraction up to 1 ends in the residual fraction send to incineration. As an example the total MRF recovery efficiency for PET bottles, for a low performing MRF producing few MRF outputs, is $0.40+0.03+0.32=0.75$. Consequently, $75 \%$ of the PET bottles is recovered $(40 \%$ in the PET output, $3 \%$ in the HDPE output and $32 \%$ in the mixed plastic fraction) for low performing MRFs producing few outputs, whereas the remaining $25 \%$ ends in the residual fraction.

Recovery efficiencies of the target materials that are based on primary literature are highlighted in bold. The other sorting efficiencies are estimated as explained in section S1.3.2. 


\section{S1.3.1.1: To PET bottles, HDPE bottles and mixed plastics}

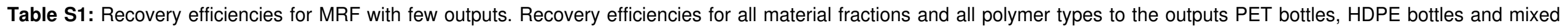
plastic.

\begin{tabular}{|c|c|c|c|c|c|c|c|c|c|c|c|c|c|c|c|c|c|c|}
\hline \multicolumn{19}{|c|}{ To PET bottles } \\
\hline MRF performance & \multicolumn{6}{|c|}{ Low } & \multicolumn{6}{|c|}{ Average } & \multicolumn{6}{|c|}{ High } \\
\hline & PET & HDPE & LDPE & PP & PS & Others & PET & HDPE & LDPE & PP & PS & Others & PET & HDPE & LDPE & PP & PS & Others \\
\hline Bottles & 0.40 & 0.10 & 0.10 & 0.10 & 0.10 & 0.10 & 0.68 & 0.07 & 0.07 & 0.07 & 0.07 & 0.07 & 0.95 & 0.03 & 0.03 & 0.03 & 0.03 & 0.03 \\
\hline Hard packaging & 0.01 & 0.10 & 0.10 & 0.10 & 0.10 & 0.10 & 0.01 & 0.07 & 0.07 & 0.07 & 0.07 & 0.07 & 0.01 & 0.03 & 0.03 & 0.03 & 0.03 & 0.03 \\
\hline Soft packaging & 0.10 & 0.10 & 0.10 & 0.10 & 0.10 & 0.10 & 0.07 & 0.07 & 0.06 & 0.07 & 0.07 & 0.07 & 0.03 & 0.03 & 0.02 & 0.03 & 0.03 & 0.03 \\
\hline Other plastic items & 0.10 & 0.10 & 0.10 & 0.10 & 0.10 & 0.10 & 0.28 & 0.07 & 0.07 & 0.07 & 0.07 & 0.07 & 0.45 & 0.03 & 0.03 & 0.03 & 0.03 & 0.03 \\
\hline Non-plastic items & 0.10 & 0.10 & 0.10 & 0.10 & 0.10 & 0.10 & 0.07 & 0.07 & 0.07 & 0.07 & 0.07 & 0.07 & 0.03 & 0.03 & 0.03 & 0.03 & 0.03 & 0.03 \\
\hline \multicolumn{19}{|c|}{ To HDPE bottles } \\
\hline MRF performance & \multicolumn{6}{|c|}{ Low } & \multicolumn{6}{|c|}{ Average } & \multicolumn{6}{|c|}{ High } \\
\hline & PET & HDPE & LDPE & PP & PS & Others & PET & HDPE & LDPE & $\mathrm{PP}$ & PS & Others & PET & HDPE & LDPE & $\mathrm{PP}$ & PS & Others \\
\hline Bottles & 0.03 & 0.35 & 0.03 & 0.03 & 0.03 & 0.03 & 0.02 & 0.63 & 0.02 & 0.02 & 0.02 & 0.02 & 0.01 & 0.90 & 0.01 & 0.01 & 0.01 & 0.01 \\
\hline Hard packaging & 0.03 & 0.10 & 0.03 & 0.03 & 0.03 & 0.03 & 0.02 & 0.05 & 0.02 & 0.02 & 0.02 & 0.02 & 0.01 & 0.01 & 0.01 & 0.01 & 0.01 & 0.01 \\
\hline Soft packaging & 0.03 & 0.03 & 0.03 & 0.03 & 0.03 & 0.03 & 0.01 & 0.01 & 0.01 & 0.01 & 0.01 & 0.01 & 0.00 & 0.00 & 0.00 & 0.00 & 0.00 & 0.00 \\
\hline Other plastic items & 0.03 & 0.03 & 0.03 & 0.03 & 0.03 & 0.03 & 0.02 & 0.21 & 0.02 & 0.02 & 0.02 & 0.02 & 0.01 & 0.40 & 0.01 & 0.01 & 0.01 & 0.01 \\
\hline Non-plastic items & 0.03 & 0.03 & 0.03 & 0.03 & 0.03 & 0.03 & 0.01 & 0.03 & 0.01 & 0.01 & 0.01 & 0.01 & 0.00 & 0.03 & 0.00 & 0.00 & 0.00 & 0.00 \\
\hline \multicolumn{19}{|c|}{ To mix } \\
\hline MRF performance & \multicolumn{6}{|c|}{ Low } & \multicolumn{6}{|c|}{ Average } & \multicolumn{6}{|c|}{ High } \\
\hline & PET & HDPE & LDPE & $\mathrm{PP}$ & PS & Others & PET & HDPE & LDPE & $\mathrm{PP}$ & PS & Others & PET & HDPE & LDPE & PP & PS & Others \\
\hline Bottles & 0.32 & 0.32 & 0.32 & 0.56 & 0.81 & 0.47 & 0.18 & 0.18 & 0.18 & 0.73 & 0.78 & 0.26 & 0.04 & 0.04 & 0.04 & 0.89 & 0.74 & 0.06 \\
\hline Hard packaging & 0.21 & 0.02 & 0.02 & 0.26 & 0.27 & 0.02 & 0.57 & 0.02 & 0.03 & 0.58 & 0.51 & 0.04 & 0.92 & 0.03 & 0.05 & 0.89 & 0.75 & 0.07 \\
\hline Soft packaging & 0.02 & 0.02 & 0.21 & 0.26 & 0.02 & 0.02 & 0.03 & 0.03 & 0.59 & 0.15 & 0.03 & 0.19 & 0.04 & 0.04 & 0.96 & 0.04 & 0.04 & 0.35 \\
\hline Other plastic items & 0.02 & 0.02 & 0.02 & 0.26 & 0.27 & 0.02 & 0.17 & 0.17 & 0.17 & 0.49 & 0.47 & 0.17 & 0.32 & 0.32 & 0.32 & 0.71 & 0.67 & 0.32 \\
\hline Non-plastic items & 0.50 & 0.50 & 0.50 & 0.50 & 0.50 & 0.50 & 0.27 & 0.27 & 0.27 & 0.27 & 0.27 & 0.27 & 0.03 & 0.03 & 0.03 & 0.03 & 0.03 & 0.03 \\
\hline
\end{tabular}


2018 Journal of Industrial Ecology - www.wileyonlinelibrary.com/journal/iie

S1.3.1.2: To PET, HDPE, PP and mixed plastic

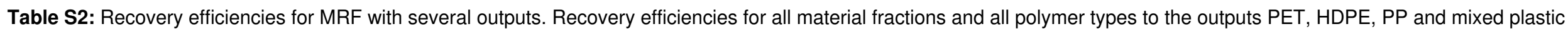

\begin{tabular}{|c|c|c|c|c|c|c|c|c|c|c|c|c|c|c|c|c|c|c|}
\hline \multicolumn{19}{|c|}{ To PET bottles } \\
\hline MRF performance & \multicolumn{6}{|c|}{ Low } & \multicolumn{6}{|c|}{ Average } & \multicolumn{6}{|c|}{ High } \\
\hline & PET & HDPE & LDPE & $\mathrm{PP}$ & PS & Others & PET & HDPE & LDPE & $\mathrm{PP}$ & PS & Others & PET & HDPE & LDPE & $\mathrm{PP}$ & PS & Others \\
\hline Bottles & 0.40 & 0.10 & 0.10 & 0.10 & 0.10 & 0.10 & 0.68 & 0.07 & 0.07 & 0.07 & 0.07 & 0.07 & 0.95 & 0.03 & 0.03 & 0.03 & 0.03 & 0.03 \\
\hline Hard packaging & 0.20 & 0.10 & 0.10 & 0.10 & 0.10 & 0.10 & 0.55 & 0.07 & 0.07 & 0.07 & 0.07 & 0.07 & 0.90 & 0.03 & 0.03 & 0.03 & 0.03 & 0.03 \\
\hline Soft packaging & 0.10 & 0.10 & 0.10 & 0.10 & 0.10 & 0.10 & 0.07 & 0.07 & 0.07 & 0.07 & 0.07 & 0.07 & 0.03 & 0.03 & 0.03 & 0.03 & 0.03 & 0.03 \\
\hline Other plastic items & 0.15 & 0.10 & 0.10 & 0.10 & 0.10 & 0.10 & 0.53 & 0.07 & 0.07 & 0.07 & 0.07 & 0.07 & 0.90 & 0.03 & 0.03 & 0.03 & 0.03 & 0.03 \\
\hline Non-plastic items & 0.10 & 0.10 & 0.10 & 0.10 & 0.10 & 0.10 & 0.07 & 0.07 & 0.07 & 0.07 & 0.07 & 0.07 & 0.03 & 0.03 & 0.03 & 0.03 & 0.03 & 0.03 \\
\hline \multicolumn{19}{|c|}{ To HDPE bottles } \\
\hline MRF performance & \multicolumn{6}{|c|}{ Low } & \multicolumn{6}{|c|}{ Average } & \multicolumn{6}{|c|}{ High } \\
\hline & PET & HDPE & LDPE & PP & PS & Others & PET & HDPE & LDPE & PP & PS & Others & PET & HDPE & LDPE & PP & PS & Others \\
\hline Bottles & 0.03 & 0.35 & 0.03 & 0.03 & 0.03 & 0.03 & 0.02 & 0.63 & 0.02 & 0.02 & 0.02 & 0.02 & 0.01 & 0.90 & 0.01 & 0.01 & 0.01 & 0.01 \\
\hline Hard packaging & 0.03 & 0.15 & 0.03 & 0.03 & 0.03 & 0.03 & 0.02 & 0.50 & 0.02 & 0.02 & 0.02 & 0.02 & 0.01 & 0.85 & 0.01 & 0.01 & 0.01 & 0.01 \\
\hline Soft packaging & 0.03 & 0.03 & 0.03 & 0.03 & 0.03 & 0.03 & 0.01 & 0.01 & 0.01 & 0.01 & 0.01 & 0.01 & 0.00 & 0.00 & 0.00 & 0.00 & 0.00 & 0.00 \\
\hline Other plastic items & 0.03 & 0.03 & 0.03 & 0.03 & 0.03 & 0.03 & 0.02 & 0.02 & 0.02 & 0.02 & 0.02 & 0.02 & 0.01 & 0.01 & 0.01 & 0.01 & 0.01 & 0.01 \\
\hline Non-plastic items & 0.03 & 0.03 & 0.03 & 0.03 & 0.03 & 0.03 & 0.01 & 0.03 & 0.01 & 0.01 & 0.01 & 0.01 & 0.00 & 0.03 & 0.00 & 0.00 & 0.00 & 0.00 \\
\hline \multicolumn{19}{|c|}{ To PP } \\
\hline MRF performance & \multicolumn{6}{|c|}{ Low } & \multicolumn{6}{|c|}{ Average } & \multicolumn{6}{|c|}{ High } \\
\hline & PET & HDPE & LDPE & PP & PS & Others & PET & HDPE & LDPE & PP & PS & Others & PET & HDPE & LDPE & $\mathrm{PP}$ & PS & Others \\
\hline Bottles & 0.01 & 0.01 & 0.01 & 0.25 & 0.01 & 0.01 & 0.00 & 0.00 & 0.00 & 0.55 & 0.00 & 0.00 & 0.00 & 0.00 & 0.00 & 0.85 & 0.00 & 0.00 \\
\hline Hard packaging & 0.01 & 0.01 & 0.01 & 0.25 & 0.01 & 0.01 & 0.01 & 0.01 & 0.01 & 0.55 & 0.01 & 0.01 & 0.01 & 0.01 & 0.01 & 0.85 & 0.01 & 0.01 \\
\hline Soft packaging & 0.01 & 0.01 & 0.01 & 0.25 & 0.01 & 0.01 & 0.00 & 0.00 & 0.00 & 0.13 & 0.00 & 0.00 & 0.00 & 0.00 & 0.00 & 0.00 & 0.00 & 0.00 \\
\hline Other plastic items & 0.01 & 0.01 & 0.01 & 0.25 & 0.01 & 0.01 & 0.01 & 0.01 & 0.01 & 0.13 & 0.01 & 0.01 & 0.01 & 0.01 & 0.01 & 0.01 & 0.01 & 0.01 \\
\hline Non-plastic items & 0.01 & 0.01 & 0.01 & 0.01 & 0.01 & 0.01 & 0.01 & 0.01 & 0.01 & 0.01 & 0.01 & 0.01 & 0.01 & 0.01 & 0.01 & 0.01 & 0.01 & 0.01 \\
\hline \multicolumn{19}{|c|}{ To mix } \\
\hline MRF performance & \multicolumn{6}{|c|}{ Low } & \multicolumn{6}{|c|}{ Average } & \multicolumn{6}{|c|}{ High } \\
\hline & PET & HDPE & LDPE & PP & PS & Others & PET & HDPE & LDPE & $\mathrm{PP}$ & PS & Others & PET & HDPE & LDPE & $\mathrm{PP}$ & PS & Others \\
\hline Bottles & 0.31 & 0.31 & 0.31 & 0.31 & 0.56 & 0.46 & 0.17 & 0.17 & 0.17 & 0.17 & 0.65 & 0.26 & 0.04 & 0.04 & 0.04 & 0.04 & 0.74 & 0.06 \\
\hline Hard packaging & 0.31 & 0.31 & 0.31 & 0.31 & 0.56 & 0.46 & 0.17 & 0.17 & 0.17 & 0.17 & 0.65 & 0.26 & 0.04 & 0.04 & 0.04 & 0.04 & 0.74 & 0.06 \\
\hline Soft packaging & 0.31 & 0.31 & 0.40 & 0.31 & 0.31 & 0.46 & 0.17 & 0.17 & 0.68 & 0.17 & 0.17 & 0.40 & 0.04 & 0.04 & 0.96 & 0.04 & 0.04 & 0.35 \\
\hline Other plastic items & 0.46 & 0.46 & 0.46 & 0.46 & 0.71 & 0.46 & 0.38 & 0.38 & 0.38 & 0.38 & 0.68 & 0.38 & 0.31 & 0.31 & 0.31 & 0.31 & 0.66 & 0.31 \\
\hline Non-plastic items & 0.50 & 0.50 & 0.50 & 0.50 & 0.50 & 0.50 & 0.27 & 0.27 & 0.27 & 0.27 & 0.27 & 0.27 & 0.03 & 0.03 & 0.03 & 0.03 & 0.03 & 0.03 \\
\hline
\end{tabular}


2018 Journal of Industrial Ecology - www.wileyonlinelibrary.com/journal/iie

\section{S1.3.1.3: To PET, HDPE, PP, PS, Film and mixed plastic}

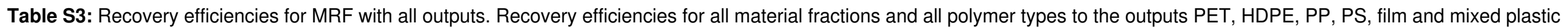

\begin{tabular}{|c|c|c|c|c|c|c|c|c|c|c|c|c|c|c|c|c|c|c|}
\hline \multicolumn{19}{|c|}{ To PET } \\
\hline MRF performance & \multicolumn{6}{|c|}{ Low } & \multicolumn{6}{|c|}{ Average } & \multicolumn{6}{|c|}{ High } \\
\hline & PET & HDPE & LDPE & PP & PS & Others & PET & HDPE & LDPE & PP & PS & Others & PET & HDPE & LDPE & PP & PS & Others \\
\hline Bottles & 0.40 & 0.10 & 0.10 & 0.10 & 0.10 & 0.10 & 0.68 & 0.07 & 0.07 & 0.07 & 0.07 & 0.07 & 0.95 & 0.03 & 0.03 & 0.03 & 0.03 & 0.03 \\
\hline Hard packaging & 0.20 & 0.10 & 0.10 & 0.10 & 0.10 & 0.10 & 0.55 & 0.07 & 0.07 & 0.07 & 0.07 & 0.07 & 0.90 & 0.03 & 0.03 & 0.03 & 0.03 & 0.03 \\
\hline Soft packaging & 0.10 & 0.10 & 0.10 & 0.10 & 0.10 & 0.10 & 0.07 & 0.07 & 0.07 & 0.07 & 0.07 & 0.07 & 0.03 & 0.03 & 0.03 & 0.03 & 0.03 & 0.03 \\
\hline Other plastic items & 0.15 & 0.10 & 0.10 & 0.10 & 0.10 & 0.10 & 0.53 & 0.07 & 0.07 & 0.07 & 0.07 & 0.07 & 0.90 & 0.03 & 0.03 & 0.03 & 0.03 & 0.03 \\
\hline Non-plastic items & 0.10 & 0.10 & 0.10 & 0.10 & 0.10 & 0.10 & 0.07 & 0.07 & 0.07 & 0.07 & 0.07 & 0.07 & 0.03 & 0.03 & 0.03 & 0.03 & 0.03 & 0.03 \\
\hline \multicolumn{19}{|c|}{ To HDPE } \\
\hline MRF performance & \multicolumn{6}{|c|}{ Low } & \multicolumn{6}{|c|}{ Average } & \multicolumn{6}{|c|}{ High } \\
\hline & PET & HDPE & LDPE & & PS & Others & PET & HDPE & LDPE & PP & PS & Others & PET & HDPE & LDPE & PP & PS & Others \\
\hline Bottles & 0.03 & 0.35 & 0.03 & 0.03 & 0.03 & 0.03 & 0.02 & 0.63 & 0.02 & 0.02 & 0.02 & 0.02 & 0.01 & 0.90 & 0.01 & 0.01 & 0.01 & 0.01 \\
\hline Hard packaging & 0.03 & 0.15 & 0.03 & 0.03 & 0.03 & 0.03 & 0.02 & 0.50 & 0.02 & 0.02 & 0.02 & 0.02 & 0.01 & 0.85 & 0.01 & 0.01 & 0.01 & 0.01 \\
\hline Soft packaging & 0.03 & 0.03 & 0.03 & 0.03 & 0.03 & 0.03 & 0.01 & 0.01 & 0.01 & 0.01 & 0.01 & 0.01 & 0.00 & 0.00 & 0.00 & 0.00 & 0.00 & 0.00 \\
\hline Other plastic items & 0.03 & 0.03 & 0.03 & 0.03 & 0.03 & 0.03 & 0.02 & 0.02 & 0.02 & 0.02 & 0.02 & 0.02 & 0.01 & 0.01 & 0.01 & 0.01 & 0.01 & 0.01 \\
\hline Non-plastic items & 0.03 & 0.03 & 0.03 & 0.03 & 0.03 & 0.03 & 0.01 & 0.03 & 0.01 & 0.01 & 0.01 & 0.01 & 0.00 & 0.03 & 0.00 & 0.00 & 0.00 & 0.00 \\
\hline \multicolumn{19}{|c|}{ To PP } \\
\hline MRF performance & \multicolumn{6}{|c|}{ Low } & \multicolumn{6}{|c|}{ Average } & \multicolumn{6}{|c|}{ High } \\
\hline & PET & HDPE & LDPE & & PS & Others & PET & HDPE & LDPE & $\mathrm{PP}$ & PS & Others & PET & HDPE & LDPE & $\mathrm{PP}$ & PS & Others \\
\hline Bottles & 0.01 & 0.01 & 0.01 & 0.25 & 0.01 & 0.01 & 0.00 & 0.00 & 0.00 & 0.55 & 0.00 & 0.00 & 0.00 & 0.00 & 0.00 & 0.85 & 0.00 & 0.00 \\
\hline Hard packaging & 0.01 & 0.01 & 0.01 & 0.25 & 0.01 & 0.01 & 0.01 & 0.01 & 0.01 & 0.55 & 0.01 & 0.01 & 0.01 & 0.01 & 0.01 & 0.85 & 0.01 & 0.01 \\
\hline Soft packaging & 0.01 & 0.01 & 0.01 & 0.25 & 0.01 & 0.01 & 0.00 & 0.00 & 0.00 & 0.13 & 0.00 & 0.00 & 0.00 & 0.00 & 0.00 & 0.00 & 0.00 & 0.00 \\
\hline Other plastic items & 0.01 & 0.01 & 0.01 & 0.25 & 0.01 & 0.01 & 0.01 & 0.01 & 0.01 & 0.13 & 0.01 & 0.01 & 0.01 & 0.01 & 0.01 & 0.01 & 0.01 & 0.01 \\
\hline Non-plastic items & 0.01 & 0.01 & 0.01 & 0.01 & 0.01 & 0.01 & 0.01 & 0.01 & 0.01 & 0.01 & 0.01 & 0.01 & 0.01 & 0.01 & 0.01 & 0.01 & 0.01 & 0.01 \\
\hline \multicolumn{19}{|c|}{ To PS } \\
\hline MRF performance & \multicolumn{6}{|c|}{ Low } & \multicolumn{6}{|c|}{ Average } & \multicolumn{6}{|c|}{ High } \\
\hline & PET & HDPE & LDPE & PP & PS & Others & PET & HDPE & LDPE & PP & PS & Others & PET & HDPE & LDPE & PP & PS & Others \\
\hline Bottles & 0.00 & 0.00 & 0.00 & 0.00 & 0.25 & 0.00 & 0.00 & 0.00 & 0.00 & 0.00 & 0.48 & 0.00 & 0.00 & 0.00 & 0.00 & 0.00 & 0.70 & 0.00 \\
\hline Hard packaging & 0.00 & 0.00 & 0.00 & 0.00 & 0.25 & 0.00 & 0.00 & 0.00 & 0.00 & 0.00 & 0.48 & 0.00 & 0.00 & 0.00 & 0.00 & 0.00 & 0.70 & 0.00 \\
\hline Soft packaging & 0.00 & 0.00 & 0.00 & 0.00 & 0.00 & 0.00 & 0.00 & 0.00 & 0.00 & 0.00 & 0.00 & 0.00 & 0.00 & 0.00 & 0.00 & 0.00 & 0.00 & 0.00 \\
\hline Other plastic items & 0.00 & 0.00 & 0.00 & 0.00 & 0.25 & 0.00 & 0.00 & 0.00 & 0.00 & 0.00 & 0.48 & 0.00 & 0.00 & 0.00 & 0.00 & 0.00 & 0.70 & 0.00 \\
\hline Non-plastic items & 0.00 & 0.00 & 0.00 & 0.00 & 0.00 & 0.00 & 0.00 & 0.00 & 0.00 & 0.00 & 0.00 & 0.00 & 0.00 & 0.00 & 0.00 & 0.00 & 0.00 & 0.00 \\
\hline
\end{tabular}


2018 Journal of Industrial Ecology - www.wileyonlinelibrary.com/journal/iie

Table S3 (continued): Recovery efficiencies for all material fractions and all polymer types to the outputs PET, HDPE, PP, PS, film and mixed plastic

\begin{tabular}{|c|c|c|c|c|c|c|c|c|c|c|c|c|c|c|c|c|c|c|}
\hline \multicolumn{19}{|c|}{ To Film } \\
\hline MRF performance & \multicolumn{6}{|c|}{ Low } & \multicolumn{6}{|c|}{ Average } & \multicolumn{6}{|c|}{ High } \\
\hline & PET & HDPE & LDPE & PP & PS & Others & PET & HDPE & LDPE & PP & PS & Others & PET & HDPE & LDPE & PP & PS & Others \\
\hline Bottles & 0.01 & 0.01 & 0.01 & 0.01 & 0.01 & 0.01 & 0.01 & 0.01 & 0.01 & 0.01 & 0.01 & 0.01 & 0.01 & 0.01 & 0.01 & 0.01 & 0.01 & 0.01 \\
\hline Hard packaging & 0.01 & 0.01 & 0.01 & 0.01 & 0.01 & 0.01 & 0.01 & 0.01 & 0.01 & 0.01 & 0.01 & 0.01 & 0.01 & 0.01 & 0.01 & 0.01 & 0.01 & 0.01 \\
\hline Soft packaging & 0.01 & 0.01 & 0.10 & 0.01 & 0.01 & 0.01 & 0.01 & 0.01 & 0.53 & 0.01 & 0.01 & 0.01 & 0.01 & 0.01 & 0.95 & 0.01 & 0.01 & 0.01 \\
\hline Other plastic items & 0.01 & 0.01 & 0.01 & 0.01 & 0.01 & 0.01 & 0.01 & 0.01 & 0.01 & 0.01 & 0.01 & 0.01 & 0.01 & 0.01 & 0.01 & 0.01 & 0.01 & 0.01 \\
\hline Non-plastic items & 0.01 & 0.01 & 0.01 & 0.01 & 0.01 & 0.01 & 0.01 & 0.01 & 0.01 & 0.01 & 0.01 & 0.01 & 0.01 & 0.01 & 0.01 & 0.01 & 0.01 & 0.01 \\
\hline \multicolumn{19}{|c|}{ To mix } \\
\hline MRF performance & \multicolumn{6}{|c|}{ Low } & \multicolumn{6}{|c|}{ Average } & \multicolumn{6}{|c|}{ High } \\
\hline & PET & HDPE & LDPE & $\mathrm{PP}$ & PS & Others & PET & HDPE & LDPE & $\mathrm{PP}$ & PS & Others & PET & HDPE & LDPE & PP & PS & Others \\
\hline Bottles & 0.30 & 0.30 & 0.30 & 0.30 & 0.30 & 0.45 & 0.17 & 0.17 & 0.17 & 0.17 & 0.17 & 0.25 & 0.03 & 0.03 & 0.03 & 0.03 & 0.03 & 0.05 \\
\hline Hard packaging & 0.30 & 0.30 & 0.30 & 0.30 & 0.30 & 0.45 & 0.17 & 0.17 & 0.17 & 0.17 & 0.17 & 0.25 & 0.03 & 0.03 & 0.03 & 0.03 & 0.03 & 0.05 \\
\hline Soft packaging & 0.30 & 0.30 & 0.30 & 0.30 & 0.30 & 0.45 & 0.17 & 0.17 & 0.16 & 0.17 & 0.17 & 0.25 & 0.03 & 0.03 & 0.01 & 0.03 & 0.03 & 0.05 \\
\hline Other plastic items & 0.45 & 0.45 & 0.45 & 0.45 & 0.45 & 0.45 & 0.38 & 0.38 & 0.38 & 0.38 & 0.38 & 0.38 & 0.30 & 0.30 & 0.30 & 0.30 & 0.30 & 0.30 \\
\hline Non-plastic items & 0.50 & 0.50 & 0.50 & 0.50 & 0.50 & 0.50 & 0.27 & 0.27 & 0.27 & 0.27 & 0.27 & 0.27 & 0.03 & 0.03 & 0.03 & 0.03 & 0.03 & 0.03 \\
\hline
\end{tabular}




\section{S1.3.2: Estimation of efficiencies for non-target materials}

Data related to sorting efficiencies of non-target materials are very limited and for most material fractions and outputs completely missing in literature, making an estimation of these necessary. This estimation was done using the following procedure:

1. Maximum and minimum concentration levels for the outputs PET, HDPE, PP, PS, Film and mixed plastic were identified based on reported concentrations from ÆErenlund (2016), RRS (2015), Luijsterburg and Goossens (2014), Jansen et al. (2012) and Enviros (2009), as illustrated in Figure S1a-f.

2. Maximum and minimum sorting efficiencies for non-target material fractions were defined and adjusted until the concentration of the MRF outputs in most of the scenarios lied within the concentration limits and still represents the range of concentrations reported in literature (see Figure S1a-f).

3. The recovery efficiencies for the average scenarios were always an average between the corresponding recovery efficiency in the high and low performing scenarios.

From the figure it can be seen that the concentration of the PET, HDPE, PP, PS and film outputs in most of the scenarios were within these limits, however, for the mixed plastic only one third of the scenarios had outputs with concentrations within the given limits (one third above the maximum and one third below the minimum). Nevertheless, taking into consideration that the limits were based on a very limited number of studies, and thereby a very limited number of actual MRFs or laboratory set-ups, a wider spread in concentration is in reality expected, having in mind that 97 sorting facilities treating plastic packaging exist solely in Spain (Janesen et al., 2013). Moreover, the minimum concentration value, taken from Enviros (2009), describes and average purity of the mixed plastic fraction from several different MRFs, without an indication of the deviation. It is therefore realistic to assume that this limit in reality is below the one reported in Enviros (2009). Consequently, the concentrations modelled in this study was found realistic, having in mind that this study aims to model the entire range of possible plastic recycling systems in Europe, from the ultimately best one to the ultimately worst. 

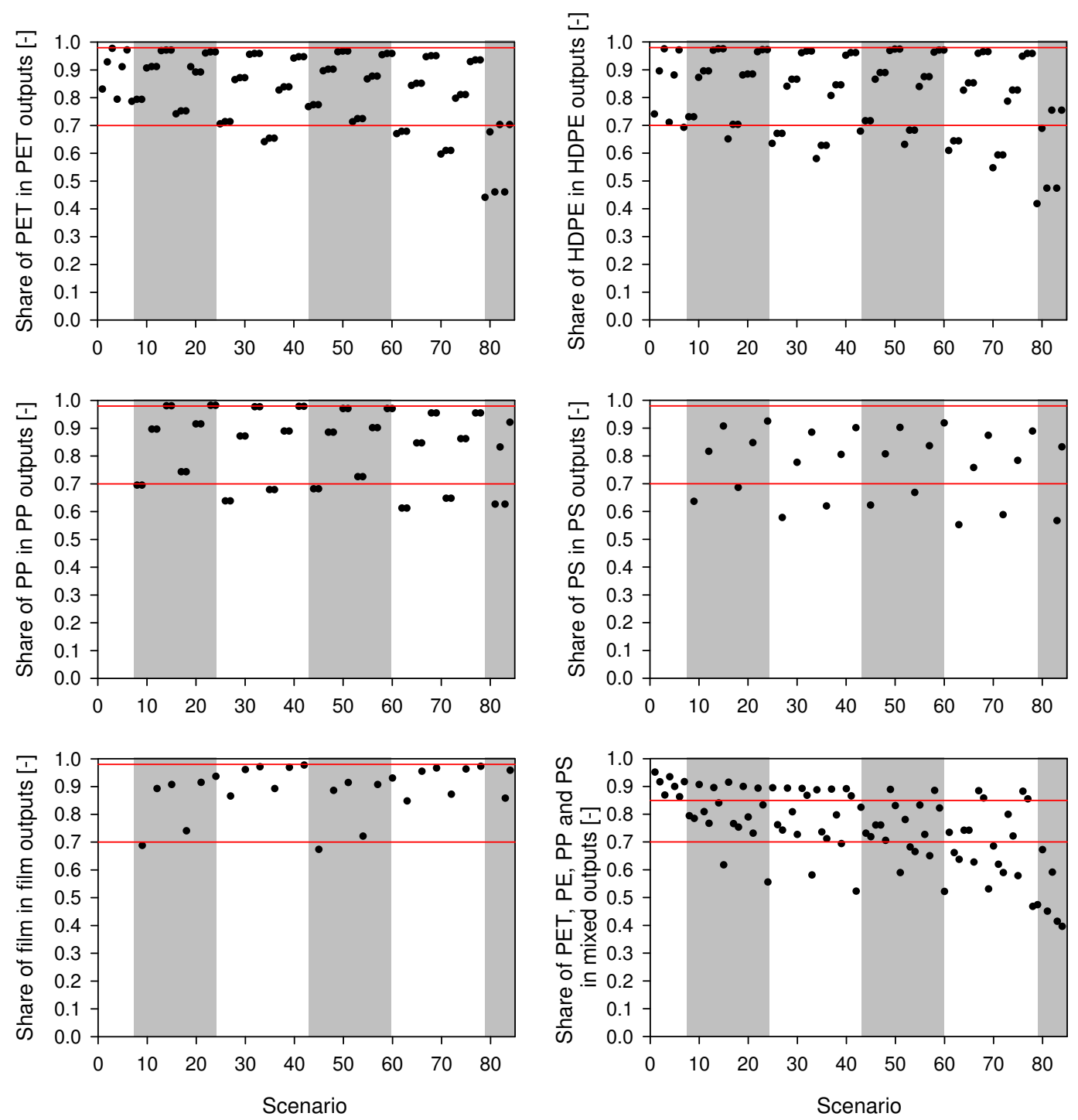

Figure S1: Concentration ranges as found in literature in red and concentration of a) PET, b) HDPE, c) PP, d) PS, e) film and d) mixed plastic outputs from the 84 different scenarios.

\section{S1.4: Outputs}

The final scenario step was related to the number and types of outputs produced from the MRF. Three choices were identified.

The few outputs choice was the simplest one where only PET and HDPE bottles were sorted into mono-polymer streams. Many UK and US MRFs treating comingled recyclables follow this approach (Enviros, 2009; RRS, 2015). The several outputs choice included the production of the most common mono-polymer streams, i.e. PET, HDPE and PP. The all possible outputs choice represented the most advanced kind of MRF producing the highest possible number of outputs, which according to Cimpan et al. (2015) includes the polymer types PET, PE, PP and PS. Thus, it included the production of PET, HDPE, PP, PS and film (mainly 
2018 Journal of Industrial Ecology - www.wileyonlinelibrary.com/journal/iie

LDPE). Additionally, the production of a residual stream and a stream of mixed plastic were included in all scenarios. 


\section{S1.5: Scenario list}

Table S4: Complete list of all 84 scenarios

\begin{tabular}{|c|c|c|c|c|}
\hline$\overline{\text { Scenario\# }}$ & Sorting scheme & $\begin{array}{l}\text { Collection } \\
\text { eff. }\end{array}$ & MRF eff. & Outputs \\
\hline 1 & Bottles & Low & Low & Few \\
\hline 2 & Bottles & Low & Average & Few \\
\hline 3 & Bottles & Low & High & Few \\
\hline 4 & Bottles & High & Low & Few \\
\hline 5 & Bottles & High & Average & Few \\
\hline 6 & Bottles & High & High & Few \\
\hline 7 & Rigid plastic & Low & Low & Few \\
\hline 8 & Rigid plastic & Low & Low & Several \\
\hline 9 & Rigid plastic & Low & Low & All possible \\
\hline 10 & Rigid plastic & Low & Average & Few \\
\hline 11 & Rigid plastic & Low & Average & Several \\
\hline 12 & Rigid plastic & Low & Average & All possible \\
\hline 13 & Rigid plastic & Low & High & Few \\
\hline 14 & Rigid plastic & Low & High & Several \\
\hline 15 & Rigid plastic & Low & High & All possible \\
\hline 16 & Rigid plastic & High & Low & Few \\
\hline 17 & Rigid plastic & High & Low & Several \\
\hline 18 & Rigid plastic & High & Low & All possible \\
\hline 19 & Rigid plastic & High & Average & Few \\
\hline 20 & Rigid plastic & High & Average & Several \\
\hline 21 & Rigid plastic & High & Average & All possible \\
\hline 22 & Rigid plastic & High & High & Few \\
\hline 23 & Rigid plastic & High & High & Several \\
\hline 24 & Rigid plastic & High & High & All possible \\
\hline 25 & Rigid and foil & Low & Low & Few \\
\hline 26 & Rigid and foil & Low & Low & Several \\
\hline 27 & Rigid and foil & Low & Low & All possible \\
\hline 28 & Rigid and foil & Low & Average & Few \\
\hline 29 & Rigid and foil & Low & Average & Several \\
\hline 30 & Rigid and foil & Low & Average & All possible \\
\hline 31 & Rigid and foil & Low & High & Few \\
\hline 32 & Rigid and foil & Low & High & Several \\
\hline 33 & Rigid and foil & Low & High & All possible \\
\hline 34 & Rigid and foil & High & Low & Few \\
\hline 35 & Rigid and foil & High & Low & Several \\
\hline 36 & Rigid and foil & High & Low & All possible \\
\hline
\end{tabular}


Table S4 (continued): Complete list of all 84 scenarios

\begin{tabular}{|c|c|c|c|c|}
\hline Scenario\# & Sorting scheme & $\begin{array}{l}\text { Collection } \\
\text { eff. }\end{array}$ & MRF eff. & Outputs \\
\hline 37 & Rigid and foil & High & Average & Few \\
\hline 38 & Rigid and foil & High & Average & Several \\
\hline 39 & Rigid and foil & High & Average & All possible \\
\hline 40 & Rigid and foil & High & High & Few \\
\hline 41 & Rigid and foil & High & High & Several \\
\hline 42 & Rigid and foil & High & High & All possible \\
\hline 43 & Containers & Low & Low & Few \\
\hline 44 & Containers & Low & Low & Several \\
\hline 45 & Containers & Low & Low & All possible \\
\hline 46 & Containers & Low & Average & Few \\
\hline 47 & Containers & Low & Average & Several \\
\hline 48 & Containers & Low & Average & All possible \\
\hline 49 & Containers & Low & High & Few \\
\hline 50 & Containers & Low & High & Several \\
\hline 51 & Containers & Low & High & All possible \\
\hline 52 & Containers & High & Low & Few \\
\hline 53 & Containers & High & Low & Several \\
\hline 54 & Containers & High & Low & All possible \\
\hline 55 & Containers & High & Average & Few \\
\hline 56 & Containers & High & Average & Several \\
\hline 57 & Containers & High & Average & All possible \\
\hline 58 & Containers & High & High & Few \\
\hline 59 & Containers & High & High & Several \\
\hline 60 & Containers & High & High & All possible \\
\hline 61 & Containers and fibres & Low & Low & Few \\
\hline 62 & Containers and fibres & Low & Low & Several \\
\hline 63 & Containers and fibres & Low & Low & All possible \\
\hline 64 & Containers and fibres & Low & Average & Few \\
\hline 65 & Containers and fibres & Low & Average & Several \\
\hline 66 & Containers and fibres & Low & Average & All possible \\
\hline 67 & Containers and fibres & Low & High & Few \\
\hline 68 & Containers and fibres & Low & High & Several \\
\hline 69 & Containers and fibres & Low & High & All possible \\
\hline 70 & Containers and fibres & High & Low & Few \\
\hline 71 & Containers and fibres & High & Low & Several \\
\hline 72 & Containers and fibres & High & Low & All possible \\
\hline 73 & Containers and fibres & High & Average & Few \\
\hline 74 & Containers and fibres & High & Average & Several \\
\hline 75 & Containers and fibres & High & Average & All possible \\
\hline
\end{tabular}


Table S4 (continued): Complete list of all 84 scenarios

\begin{tabular}{lllll}
\hline Scenario\# & Sorting scheme & $\begin{array}{l}\text { Collection } \\
\text { eff. }\end{array}$ & MRF eff. & Outputs \\
\hline 76 & Containers and fibres & High & High & Few \\
77 & Containers and fibres & High & High & Several \\
78 & Containers and fibres & High & High & All possible \\
79 & No separation & - & Low & Few \\
80 & No separation & - & Average & Few \\
81 & No separation & - & Low & Several \\
82 & No separation & - & Average & Several \\
83 & No separation & - & Low & All possible \\
84 & No separation & - & Average & All possible \\
\hline
\end{tabular}

Table S5: List of theoretical scenario configurations that were not found realistic and hence excluded from the study.

\begin{tabular}{lllll}
\hline & Sorting scheme & Collection eff. MRF eff. & Outputs \\
\hline 85 & Bottles & Low & Low & Several \\
86 & Bottles & Low & Average & Several \\
87 & Bottles & Low & High & Several \\
88 & Bottles & High & Low & Several \\
89 & Bottles & High & Average & Several \\
90 & Bottles & High & High & Several \\
91 & Bottles & Low & Low & All possible \\
92 & Bottles & Low & Average & All possible \\
93 & Bottles & Low & High & All possible \\
94 & Bottles & High & Low & All possible \\
95 & Bottles & High & Average & All possible \\
96 & Bottles & High & High & All possible \\
97 & No separation & Low & Low & Few \\
98 & No separation & Low & Average & Few \\
99 & No separation & Low & Low & Several \\
100 & No separation & Low & Average & Several \\
101 & No separation & Low & Low & All possible \\
102 & No separation & Low & Average & All possible \\
103 & No separation & High & Low & Few \\
104 & No separation & High & Average & Few \\
105 & No separation & High & Low & Several \\
106 & No separation & High & Average & Several \\
107 & No separation & High & Low & All possible \\
108 & No separation & High & Average & All possible \\
\hline
\end{tabular}




\section{Section S2: Quality assessment}

\section{S2.1: Definition of application groups}

All plastic on the European market needs to comply with the chemical requirements in REACH (EU, 2006).

Food packaging (FP) are described in Commission Regulation (EU) No $10 / 2011$ and is defined as plastic materials that is: 1 ) intended to come into contact with food, 2) in contact with food or 3) expected to come into contact with food. This includes both products exclusively made of plastics as well as multi-layered materials and plastic coatings. Not included in this application groups are rubber and silicon's. (EU, 2011b)

Toys $(T)$ are regulated in European Commission toy safety directive 2009/48/EC and toys are defined as "... products designed or intended, whether or not exclusively, for use in play by children under 14 years of age" $(E C, 2013)$.

Within this application group the sub-group toys for children under 36 month or intended to be put into the mouth exists, where additional requirements need to be fulfilled. These requirements are presented in Appendix $C$ of directive 2009/48/EC and includes all commodities produced as toys intended for children under 36 month or to be put in the mouth (Safe Toys, 2015).

The legal requirements for the application group pharmaceuticals $(\mathrm{PH})$ are presented in Council directive 93/42/EEC and includes medical devices and their accessories. In this relation medical devices refers to any instrument, apparatus, appliance, material or article intended for humans with the purpose of handling diseases, injuries and handicaps as well as related to the anatomy of a physiological process or control of conception. Accessories is defined as an article needed to be used together with a medical device in order for it to perform as intended, which is not a device in itself. (EU, 2007)

Regarding the application group electrical and electronics (EE), the legal requirements are presented in Directive 2011/65/EU and electrical and electronic equipment are defined as: "1) Large household appliances, 2) Small household appliances, 3) IT and telecommunications equipment, 4) Consumer equipment, 5) Lighting equipment, 6) Electrical and electronic tools, 7) Toys leisure and sports equipment, 8) Medical devices, 9) Monitoring and control instruments including industrial monitoring and control instruments, 10) Automatic dispensers and 11) Other electrical and electronic equipment not covered by any of the categories above" (EU, 2011c) expect for military equipment, equipment intended to be sent to space, means of transport, large stationary equipment, ect. (EU, 2011c). 
The application group building and construction $(\mathrm{BC})$ includes “... any product or kit which is produced and placed on the market for incorporation in a permanent manner in construction works or parts thereof..." (EU, 2011a).

Non-food packaging is defined as devices that is produced to contain, protect or be wrapped around other products that is not included in the food packaging applications group.

The application group automotive include all plastic items used in the construction of automotive including items for: 1) electronics and light, 2) interior, 3) Exterior, and 4) under the hood (PlasticsEurope, 2013).

The final application group Others include all kinds of plastic products that is not included in any the other application groups, such as furniture, household appliances, clothing, etc. (PlasticsEurope and EPRO, 2016).

\section{S2.2: Quality criteria and assessment}

In this section the four different types of contamination, summarised in Table S6, is described in details, including the consequence for the quality as well as how this consequence is measured.

The first type of contamination, contamination with non-plastic material, most often happens during the sorting stage, due to sorting of non-plastic products or products that is not included in the sorting scheme (e.g. products made from a mixture of different materials) or contamination stuck to the surface of the plastic products (e.g. yoghurt residues stuck to a yoghurt plastic beaker). Additionally, the efficiency of the MRF is crucial for the level of non-plastic contamination present in the sorted plastic stream send to reprocessing. Too high a concentration of nonplastic impurities represents a huge problem since it can result in a lower grade of plastic produced, lowering the price of the recycled plastic significantly (ReQIP, 2014), or in worst case make the plastic stream entirely unsuited for recycling (Cappadona, 2015, Petersen and Mayland, 2015). Moreover, Petersen and Mayland (2015) emphasize that especially contamination of organics can reduce the applicability of the recycled plastic. Since contamination of the plastic waste can happen several places in the recycling chain and affect the functionality in different ways it is necessary to distinguish between different types of contamination. 
Table S6: Brief overview of the types of contaminants affecting the applicability of MRF outputs, their origin and consequence.

\begin{tabular}{|c|c|c|}
\hline $\begin{array}{l}\text { Contami- } \\
\text { nants }\end{array}$ & Origin & Consequence \\
\hline $\begin{array}{l}\text { Non-plastic } \\
\text { materials }\end{array}$ & $\begin{array}{l}\text { - Missortings of items made } \\
\text { of or containing non-plas- } \\
\text { tic material } \\
\text { - Impurities stuck to the } \\
\text { plastic products, such as } \\
\text { organic residues }\end{array}$ & $\begin{array}{l}\text { Reducing physical and mechani- } \\
\text { cal properties. }\end{array}$ \\
\hline $\begin{array}{l}\text { Non-target } \\
\text { polymers }\end{array}$ & $\begin{array}{l}\text { - Complex design of plastic } \\
\text { products made of several } \\
\text { immiscible polymers that } \\
\text { cannot be easily } \\
\text { disassembled } \\
\text { - Wrong sorting during } \\
\text { mechanical sorting }\end{array}$ & $\begin{array}{l}\text { Reducing physical and mechani- } \\
\text { cal properties. }\end{array}$ \\
\hline $\begin{array}{l}\text { Unwanted } \\
\text { products }\end{array}$ & $\begin{array}{l}\text { - In general mechanical } \\
\text { sorting cannot differ } \\
\text { between different types of } \\
\text { products. }\end{array}$ & $\begin{array}{l}\text { As different products can have dif- } \\
\text { ferent properties, a mixture of } \\
\text { products can make it difficult to } \\
\text { control the final properties of the } \\
\text { recycled plastic exactly. }\end{array}$ \\
\hline Chemicals & $\begin{array}{l}\text { - Contamination during use } \\
\text { and waste management } \\
\text { - Reaction or degradation of } \\
\text { additives during recycling } \\
\text { forming potentially } \\
\text { hazardous compounds }\end{array}$ & $\begin{array}{l}\text { If the content of chemicals are too } \\
\text { high legal limit values (migration } \\
\text { or total content) might be ex- } \\
\text { ceeded directly limiting the ap- } \\
\text { plicability. }\end{array}$ \\
\hline
\end{tabular}

The second type, contamination with non-target polymers, furthermore influences the quality (Villanueva and Eder, 2014). Since different polymer types have different properties contamination with un-wanted polymers can cause processing problems and interrupt the structure of the recycled plastic produced, reducing its mechanical properties (Villanueva and Eder, 2014). The concentration of non-target polymers in the final polymer stream send to reprocessing depends strongly on the efficiency of the MRF. However, in regards to household waste non-target polymers are practically impossible to remove entirely, even using state-of-the-art sorting facilities, since some of the products in the waste consists of multiple polymer layers that are not easily separated (Luijsterburg and Goossens, 2014).

Even within the same polymer type, different products do not necessarily have the same physical or chemical properties. Contamination with unwanted products are therefore the third type. This is an issue for e.g. HDPE used in ottles 
(produced by blow moulding), as it has a markedly different melt index than HDPE used in products made from injection moulding (e.g. caps for bottles). Thus, even though these products are of the same polymer type they do not blend into a consistent mix reducing the ways by which the recycled HDPE can be moulded (Cornell, 2016). As a result especially PE and PP streams will have to be further separated into types of products, to reach secondary material with a high applicability (Heinzel et al., 2015). Whether such a sorting can successfully take place depends on the sorting scheme (are different types of products included), the sorting efficiencies of the different products and the MRF efficiency.

These first three contamination types are related to the physical and mechanical properties of the recycled plastic, however, it is not known exactly how they affect all the individual physical and mechanical properties, and thereby the quality. Thus, in order to "translate" knowledge about these three types of contamination to how they influence the quality, specifications from reprocessing facilities were therefore used to establish this relationship. The reprocessing facilities receive sorted plastic waste from MRFs and then further treats and upgrades it into recycled plastic. However, the reprocessing facilities cannot recycle sorted plastic waste, if it is too contaminated and there therefore specify maximum acceptable contamination levels in their specifications. These were used to compile contamination limits for both non-plastic impurities, non-target polymers and product mixtures were defined. The reprocessing facilities were divided into two kinds: 1) facilities producing food grade recycled plastic and 2) facilities not producing food grade recycled plastic. Using this division it was possible to divide the contamination limits into limits for food-grade and non-food grade recycled plastic. The concentration limits are presented in Table S7 and S8. In regards to product mixtures, it was found that the reprocessing facilities especially emphasise the sorting of bottles from other items and to a smaller extend focus on sorting other kinds of items from the stream. Table S8 is therefore divided into contamination limits for bottles and other items. 
Table S7 Limits of non-plastic contamination in the incoming waste stream received at reprocessing facilities. The main products are listed in the first left column and the contamination items are listed at the top rows. All numbers are given in weight \% of the total waste stream. The limits are defined based on CLR (2016a), CLR (2016b), DKR (2014a), DKR (2014c), DKR (2012a), DKR (2012b), DKR (2012c), DKR (2012d), APR (2013a), APR (2013b), APR (2016a), APR (2016b), APR (2016c), APR (2016d), ReQIP (2014) and Biffa Polymers (2016)

\begin{tabular}{|c|c|c|c|c|c|c|c|}
\hline & Total & Aluminium & Steel & Paper & Cardboard & Food residues & Others \\
\hline & \multicolumn{7}{|c|}{ Food grade } \\
\hline PET & $2-6$ & \multicolumn{2}{|c|}{$0.5-6$} & & -6 & 1 & 2 \\
\hline HDPE & $5-6$ & \multicolumn{2}{|c|}{$0-6$} & & -6 & 1 & 2 \\
\hline & \multicolumn{7}{|c|}{ Non-food grade } \\
\hline & \multicolumn{7}{|c|}{ All applications except food packaging } \\
\hline PET & $2-6$ & \multicolumn{2}{|c|}{$0.5-6$} & & -6 & $1-2$ & 2 \\
\hline HDPE & $2-21$ & $2-6$ & $0-6$ & & -6 & $1-2$ & 2 \\
\hline PE & $2-21$ & \multicolumn{2}{|c|}{$0.5-6$} & & -6 & $1-2$ & $2-3$ \\
\hline PP & $6-8$ & \multicolumn{2}{|c|}{$0.5-2$} & & 2 & 2 & 3 \\
\hline \multirow[t]{2}{*}{ PS } & 6 & \multicolumn{2}{|l|}{0.5} & & & 2 & 2 \\
\hline & \multicolumn{7}{|c|}{ Applications with no legal restrictions } \\
\hline Film & $5-6$ & 0.5 & & & -2 & 1 & 4 \\
\hline Mixed & $5-10$ & $1-2$ & & & -5 & 2 & $1-2$ \\
\hline
\end{tabular}


Table S8 Limits for non-target plastic contamination and product mixtures in the incoming waste stream received at reprocessing facilities. The main products are listed in the first left column and the contamination items are listed at the top rows. All numbers are given in weight \% of the total waste stream. The limits are defined based on CLR (2016a), CLR (2016b), DKR (2014a), DKR (2014c), DKR (2012a), DKR (2012b), DKR (2012c), DKR (2012d), APR (2013a), APR (2013b), APR (2016a), APR (2016b), APR (2016c), APR (2016d), ReQIP (2014) and Biffa Polymers (2016)

\begin{tabular}{|c|c|c|c|c|c|c|c|c|c|c|c|c|c|c|c|}
\hline & \multirow[b]{2}{*}{ Total } & \multicolumn{4}{|c|}{ Polymer types of bottle } & \multicolumn{10}{|c|}{ Polymer types of other items } \\
\hline & & Total & PET & HDPE & PP & Total & PET & HDPE & LDPE & PP & PS & EPS & PVC & Film & Other \\
\hline & \multicolumn{14}{|c|}{ Food grade - high quality (All applications) } & \\
\hline PET bottles & $2-10$ & $2-10$ & & $2-10$ & $2-10$ & $2-6$ & $2-6$ & $2-6$ & $2-6$ & $2-6$ & $2-6$ & 0.5 & 0.1 & $1-6$ & $2-6$ \\
\hline PET & $2-6$ & & & & & $2-6$ & 2 & & & & 2 & 0.5 & $0.1-2$ & $1-6$ & $2-6$ \\
\hline HDPE bottles & 10 & 10 & 10 & & 10 & 6 & 6 & 6 & 6 & 6 & 6 & 6 & 6 & 6 & 6 \\
\hline \multirow[t]{2}{*}{ HDPE } & $6-10$ & & & & & $6-10$ & $4-6$ & 6 & $4-6$ & $6-10$ & $4-6$ & $4-6$ & $4-6$ & $0-6$ & $4-6$ \\
\hline & \multicolumn{15}{|c|}{ Non-food grade - Medium quality (All applications except food packaging) } \\
\hline PET bottles & $2-50$ & $2-50$ & & $2-10$ & $2-10$ & $2-50$ & $2-50$ & $2-6$ & $2-6$ & $2-6$ & $2-6$ & $0.5-2$ & 0.1 & $1-6$ & $2-6$ \\
\hline PET & $2-6$ & & & & & $2-6$ & & & & & $2-6$ & $0.5-2$ & $0.1-2$ & $0-6$ & $2-6$ \\
\hline HDPE bottles & $5-21$ & $5-10$ & $5-10$ & & $5-10$ & $5-21$ & $2-6$ & $2-6$ & $2-6$ & $2-6$ & $2-6$ & $2-6$ & $0-6$ & $0-6$ & $2-6$ \\
\hline HDPE & $6-10$ & & & & & $6-10$ & $4-6$ & 6 & $4-6$ & $6-10$ & $4-6$ & $4-6$ & $4-6$ & $0-6$ & $4-6$ \\
\hline PE & $6-10$ & & & & & $6-10$ & $2-6$ & 6 & $2-6$ & $2-10$ & $2-6$ & $2-6$ & $0-6$ & $0-6$ & $2-6$ \\
\hline PP & $6-8$ & & & & & $6-20$ & 2 & $1-2$ & 1 & & 2 & 2 & 2 & $0-2$ & 3 \\
\hline \multirow[t]{2}{*}{ PS } & 6 & & & & & 6 & 4 & 4 & 4 & 4 & 4 & 4 & 4 & 1 & 4 \\
\hline & \multicolumn{15}{|c|}{ Applications with no legal restrictions - low quality } \\
\hline Film & $2-4$ & & & & & $2-4$ & $2-4$ & & & $0-4$ & $2-4$ & $2-4$ & $0-4$ & & $2-4$ \\
\hline Mixed & $5-10$ & & & & & $5-10$ & $3-5$ & $5-10$ & $5-10$ & $5-10$ & $5-10$ & $5-10$ & 0.5 & 1 & $5-10$ \\
\hline
\end{tabular}


As intervals of limits from specifications from different reprocessing facilities are given, and the waste composition in this study is not details enough to use all of the limits provided in Table S7 and S8, the average was taken and some limits were aggregated in order to use them in the study. These are given for non-plastic impurities and unwanted polymers in Table S9.

Table S9: Contamination limits for non-plastic impurities and polymer cross-contaimation for high, medium and low quality plastic. q.: quality.

\begin{tabular}{l|ccc|ccc}
\hline & \multicolumn{3}{|c|}{ Non-plastic impurities } & \multicolumn{3}{c}{ Unwanted polymers } \\
& High q. & Medium q. & Low q. & High q. & Medium q. & Low q. \\
\hline PET & 4 & 10 & 16.5 & 6 & 10 & 17 \\
HDPE & 5 & 10 & 18 & 7 & 10 & 18 \\
PP & - & 12 & 16.5 & - & 12 & 17 \\
PS & - & 5 & 7.5 & - & 5 & 8 \\
Film & - & 4.5 & 7.5 & - & 5 & 8 \\
Mix & - & - & 7.5 & - & - & 8 \\
\hline
\end{tabular}

Knowing the composition of a given MRF output, the tables were used to evaluate into what applications it can be recycled into. MRF outputs complying with limits for food-grade plastic has a potential to be used in all applications. Mono polymer MRF outputs (PET, HDPE, PP and PS) complying with limits for non-food grade plastic has a potential to be recycled into all applications expect food packaging and outputs complying with the non-food grade contamination limits for the mixed plastic fractions (film and mixed plastic) can only be recycled into low quality applications where no legal restrictions exist in relation to the chemical composition (building and construction, non-food packaging, automotive and others). This is assumed, since several studies state that mixed polymer products can only be used for low quality applications (e.g. Dvorak et al., 2009; Cimpan et al., 2015). MRF outputs where the level of contamination exceed the limits in Table S9 cannot be recycled but instead used for energy recovery.

Contamination with chemical substances represents an additional type of contamination. In production of virgin plastic, different additives are added to the pure polymer stream altering the properties of the plastic. Accordingly, when recycling plastic products with different additives, the recycled plastic material will contain an unknown concentration of additives that might exceed legal limit values for use in certain applications. Moreover, chemical contamination might also occur in the use phase of the plastic as a result of adsorption of e.g. flavourings, essential oils, etc. (Villanueva and Eder, 2014). 
Additionally, several recyclers sort the plastic according to colour, since clear plastic most often can be used in a broader range of applications and thereby have a higher price than plastic of mixed colours. However, even though a mixture of colours limits the applicability today, it might not be a limiting factor on a long term, since it is a design choice not to use mixed colours for certain application (Haupt et al., 2016). Consequently, colour contamination is not further addressed in this assessment.

Finally, several studies have suggested that the length of the polymers might be reduced during the recycling process, reducing the functionality of the recycled plastic (van der Harst et al., 2016; Rigamonti et al., 2014). However, experts state that the effect from a potential shortening in polymers due to the recycling process is very small compared to the effect from contamination with foreign material and non-target polymers (Daugaard, 2015). Consequently it is not included in the assessment.

The institutionally-prescribed functionality can additionally limit the applicability of recycled plastic. As described earlier the institutionally prescribed functionality is related to the chemical composition of recycled plastic and whether that complies with legislative requirements for use in the predefined applications. Therefore, specific European requirements to use of plastic in different applications are summarised in Table S10.

Only few studies exist related to the concentrations of the substances mentioned in Table S10 in plastic waste, reprocessed plastic or used plastic products (Pivnenko et al., 2016; Ballesteros-Gómez et al., 2014; Whitt et al., 2012; Riber et al., 2009; Ernst et al., 2000; Huber and Franz, 1997). In one of these studies the content of cadmium was found to exceed the limits for use in electrical and electronics, however this was only found in one of four TVs included in the study and none of the other electronic devices (Ernst et al., 2000). Additionally, Pivnenko et al. (2016) found that one of 7 samples of source separated household plastic waste had a concentration of DEHP exceeding the limits for toys. Nonetheless, based on existing literature no statistically convincing data were found to suspect that the limit values mentioned in Table S10 in general is exceeded for recycled plastic. However, as previously mentioned, the information in this area is scares and this conclusion might change as more information becomes available. This could, however, change if new studies related to the chemical composition of recycled plastic show different tendencies. 
Table S10 Overview of legislation in relation to use of plastic for different applications. The legislation only relates to the chemical properties of the plastic, not physical. PBB: Polybrominated bisphenyls, PBDE: Polybrominated diphenyl ethers, DBP: dibuytl phthalate, BBP: benzyl butyl phthalate, DEHP: bis (2-ethylhexyl) phthalate, CMR: Carcinogenic, mutagenic and toxic for reproduction.

\begin{tabular}{|c|c|c|}
\hline Application & Requirements & Legislation \\
\hline Food packaging & $\begin{array}{l}\text { Migration limits for } 974 \text { substances. } \\
\text { CMR classified substances should not be } \\
\text { used in food contact materials. }\end{array}$ & 2011/10/EC \\
\hline \multirow[t]{2}{*}{ Toys } & $\begin{array}{l}55 \text { allergenic fragrances cannot be used in } \\
\text { toys. } \\
\text { Migration limits for } 19 \text { substances. } \\
\text { Labelling requirements for } 11 \text { allergenic } \\
\text { fragrances. }\end{array}$ & 2009/48/EC \\
\hline & $\begin{array}{l}\text { Total limits for } 3 \text { phthalates (DBP, BBP and } \\
\text { DEHP). }\end{array}$ & EC 1907/2006 \\
\hline $\begin{array}{l}\text { Electrical and } \\
\text { electronics }\end{array}$ & $\begin{array}{l}\text { Total limits for } 6 \text { substances (Pb, Hg, Cd, } \\
\text { hexavalent } \mathrm{Cr}, \mathrm{PBB}, \mathrm{PBDE})\end{array}$ & 2011/65/EU \\
\hline Pharmaceuticals & Labelling of CMR classified phthalates & 93/42/EEC \\
\hline $\begin{array}{l}\text { Building and } \\
\text { construction }\end{array}$ & No specific legal requirements & \\
\hline $\begin{array}{l}\text { Non-food } \\
\text { packaging }\end{array}$ & No specific legal requirements & \\
\hline Automotive & No specific legal requirements & \\
\hline Others & No specific legal requirements & \\
\hline
\end{tabular}




\section{Section S3: Identification of European market shares}

The market shares presented in the main paper are mainly based on information from PlasticsEurope and EPRO (2016), where it is assumed that the market for film is equal to the market for LDPE, as most soft plastic generated in the households is expected to be LDPE. However, as PlasticsEurope and EPRO (2016) only provide information about the market shares of packaging, electrical and electronics, building and construction materials, automotive and others, additional information was needed to estimate how large a share of the packaging market is used for food or non-food contact material and how large a share of the others market that is used for toys and pharmaceuticals, within all the PET, HDPE, PP, PS, film and mixed plastic market.

\section{S3.1: Food and non-food packaging}

In the following sections it is described how the packaging fraction reported by PlasticsEurope and EPRO (2016) was divided into food and non-food packaging.

\section{S3.1.1: Film (LDPE) market}

For soft plastic, assumed to be LDPE, is was possible to find information related to the use as food and non-food packaging. According to Stubenschrott (2016) 75\% of the soft plastic packaging produced in Europe is used for food packaging, the rest for non-food packaging. This division is therefore used for the film market.

\section{S3.1.2: PET, HDPE, PP, PS market}

Such information was not available for the remaining polymer markets and the division therefore needed to be estimated. As plastic items ending up as household waste have a short lifetime it is reasonable to assume that the composition of household plastic waste is closely related to the composition of similar new plastic items produced. Therefore, household plastic waste compositions were used to estimate how large a share of plastic packaging produced for use in households that is food-contact and non-food packaging. This was done by combining information from Edjabou et al. (2015), Rigamonti et al. (2014), Petersen et al. (2014), Petersen et al. (2012) and Clemen (2014), following the given steps. 
1. In Petersen et al. (2014) and Petersen et al. (2012) compositions of plastic in Danish residual household waste are provided for single and multi-family houses with no separate collection systems for plastic. The compositions are divided into different types of plastic products. These were classified by the authors to be either food packaging or non-food packaging and belong to the waste fractions bottles, hard plastic or other plastic packaging, which are waste fractions used by Rigamonti et al. (2014) and Edjabou et al. (2016). This classification are presented in Table S11, where only the rigid plastic products are shown as data for soft plastic was already provided.

2. The different plastic product types were given a polymer composition from Edjabou et al. (2016) and Rigamonti et al. (2014) (see Table 1 in the main paper), based on the associated waste fraction (column 4 in Table S11).

3. The average composition of Danish plastic waste generated in single and multi-family houses were calculated (using equal weighting) and associated to the different polymer types.

4. The composition of the individual polymer types were then divided into food packaging or non-food packaging (based on column 3 in Table S11). From this, the total share of PET, HDPE, PP and PS generated in households previously used as food or non-food packaging was estimated and the results are presented in Table S12.

5. Based on Clemen (2014) $216 \mathrm{kt}$ plastic packaging was produced in Denmark in 2014, where $146 \mathrm{kt}$ was used as household packaging and $70 \mathrm{kt}$ as industrial packaging. Assuming that Danish conditions represents European conditions, $68 \%$ of the packaging produced in Europe is estimated to be used in households whereas the remaining $32 \%$ is estimated to be used in industry.

6. Since information related to industrial plastic waste composition is missing in literature it was assumed that $10 \%$ of the industrial packaging is used as food packaging and $90 \%$ as non-food packaging.

7. Combining all of the above information Table S13 was set up. 
Table S11: Rigid plastic product types used in the waste compositions provided in Petersen et al. (2014) and Petersen et al (2012), the previous use as food contact products (FP) or non-food contact product (NFP) and the classification of the product types into waste fractions.

\begin{tabular}{|c|c|c|c|}
\hline $\begin{array}{l}\text { Composition provided in Pe- } \\
\text { tersen et al. (2012) and Pe- } \\
\text { tersen et al. (2014) - Danish }\end{array}$ & $\begin{array}{l}\text { Composition provided in Pe- } \\
\text { tersen et al. (2012) and Petersen } \\
\text { et al. (2014) - English }\end{array}$ & Use & Waste fraction \\
\hline Plastflasker til drikkevare & Beverage plastic Bottles & FP & Bottles \\
\hline $\begin{array}{l}\text { Dunke til eddike, sprinklervæ- } \\
\text { ske ol. }\end{array}$ & $\begin{array}{l}\text { Bottles for vinegar, windshield } \\
\text { washer fluids, etc. }\end{array}$ & NFP & Bottles \\
\hline $\begin{array}{l}\text { Dunke og bøtter til } \\
\text { kemisk-tekniske produkter }\end{array}$ & $\begin{array}{l}\text { Bottles and tubs for } \\
\text { chemical-technical products }\end{array}$ & NFP & Hard packaging \\
\hline $\begin{array}{l}\text { Plastbakker til andre } \\
\text { fødevarer }\end{array}$ & $\begin{array}{l}\text { Plastic trays for other food prod- } \\
\text { ucts }\end{array}$ & FP & Hard packaging \\
\hline Urtepotter af plast & Plastic flowerpots & NFP & $\begin{array}{l}\text { Other plastic } \\
\text { items }\end{array}$ \\
\hline Plastlåg & Plastic lids & FP & Hard packaging \\
\hline Plastflasker til madvarer & Plastic Bottles for food products & FP & Bottles \\
\hline Dunke og bøtter til fødevarer & $\begin{array}{l}\text { Bottles and tubs for food prod- } \\
\text { ucts }\end{array}$ & FP & Hard packaging \\
\hline Dunke og bøtter til kemikalier & Bottles and tubs for chemicals & NFP & Hard packaging \\
\hline Plastbakker til kød & Plastic trays for meat & FP & Hard packaging \\
\hline $\begin{array}{l}\text { Plastkasser (til frugt, kager, } \\
\text { mv.) }\end{array}$ & $\begin{array}{l}\text { Plastic boxes (for fruit, cakes, } \\
\text { etc.) }\end{array}$ & FP & Hard packaging \\
\hline Anden hård emballage & Other hard plastic packaging & NFP & Hard packaging \\
\hline Blisterpakninger & Blister packs & FP & $\begin{array}{l}\text { Other plastic } \\
\text { items }\end{array}$ \\
\hline Bægre til smør og margarine & Beaker for butter & FP & Hard packaging \\
\hline Pålægspakninger & Packaging for cold cuts & FP & Hard packaging \\
\hline Plastlaminater & Plastic laminates & NFP & $\begin{array}{l}\text { Other plastic } \\
\text { items }\end{array}$ \\
\hline Bøjler & Hangers & NFP & \\
\hline Leget $\varnothing j$ & Toys & NFP & \\
\hline Køkkenting & Kitchen stuff & FP & \\
\hline Brugsgenstande & Utiliy items & NFP & Other plastic \\
\hline Blød PVC & Soft PVC & NFP & items \\
\hline Hård PVC & Hard PVC & NFP & \\
\hline Anden hård plast & Other hard plastic & NFP & \\
\hline Anden plast & Other plastic & NFP & \\
\hline
\end{tabular}


Table S12: Percentage of PET, HDPE, PP and PS waste from Danish households that was previously used as food packaging and non-food packaging.

\begin{tabular}{l|cccc}
\hline & PET & HDPE & PP & PS \\
\hline Food packaging & 81 & 75 & 66 & 63 \\
Non-food packaging & 19 & 25 & 34 & 37 \\
\hline Total & 100 & 100 & 100 & 100 \\
\hline
\end{tabular}

Table S13: Estimated percentage of PET, HDPE, PP and PS packaging produced in Europe for both household and industrial purposes that is used as food and non-food packaging.

\begin{tabular}{l|cccc}
\hline & PET & HDPE & PP & PS \\
\hline Food packaging & 58 & 54 & 48 & 46 \\
Non-food packaging & 42 & 46 & 52 & 54 \\
\hline Total & 100 & 100 & 100 & 100 \\
\hline
\end{tabular}

\section{S3.2: Toys, Pharmaceuticals and Others}

In this section it is described how the Others fraction reported by PlasticsEurope and EPRO (2016) was divided into toys, pharamaceuticals and others.

In PlasticsEurope and EPRO (2016) the European turnover of plastic was 340 billion Euros in 2015, whereas the European toy turnover was 5.6 billion Euros in 2011 (TIE, 2013). According to Baytech (2016) plastic toys are responsible for $90 \%$ of the total toy turnover. Assuming that the market for toys in Europe is the same today as in 2011 plastic toys can be estimated to represent $1.5 \%$ of the total European plastic market. Dividing this onto the markets for PET, HDPE, PP, PS and film based on their size relative to the total plastic market (given in PlasticsEurope and EPRO (2016)), the market share of plastic toys within the individual polymer markets was estimated and given in Table S14.

To estimate the size of the pharmaceutical plastic market production data were used. PlasticsEurope and EPRO (2016) state that the total European plastic production was 58 million ton in 2015. According to Eurostat (2015) the generation of "Chemicals, pharmaceuticals, rubber and plastic products"-waste was equal to 2.21 million ton. This is assumed to be equal to the amount of pharmaceutical plastic products produced in Europe and thus the total share of the pharmaceutical market can be estimated to $3.8 \%$ of the total European plastic market. As for toys this is divided onto the individual polymer markets based on their relative share of the total plastic market (given in PlasticsEurope and EPRO (2016)) and the market shares are presented in Table S14. 
The market for Others are then estimated by subtracting the market shares for toys and pharmaceuticals from the market shares of Others given by PlasticsEurope and EPRO (2016).

The market shares for mixed plastic is estimated from an average of the market shares of the other markets weighted by their relative share of the total plastic market.

Table S14: Market shares of food packaging (FP), Toys (T), Pharmaceuticals (PH), Electrical and Electronics (EE), Building and Construction (BC), Non-food packaging (NFP), Automotive (AU) and Others (OT) for the European PET, HDPE, PP, PS, Film and mixed plastic market.

\begin{tabular}{l|cccccccc}
\hline Polymer & FP & T & PH & EE & BC & NFP & AU & OT \\
\hline PET & 57.3 & 0.1 & 0.3 & 0.0 & 0.0 & 42.0 & 0.0 & 0.3 \\
Film & 54.5 & 0.3 & 0.7 & 2.6 & 6.1 & 18.2 & 1.5 & 16.3 \\
HDPE & 27.3 & 0.2 & 0.5 & 1.9 & 23.4 & 23.4 & 5.9 & 17.4 \\
PS & 15.4 & 0.1 & 0.3 & 9.7 & 42.2 & 18.1 & 0.0 & 14.2 \\
PP & 17.8 & 0.3 & 0.7 & 5.5 & 7.9 & 19.5 & 12.9 & 35.5 \\
Mixed plastic & 33.9 & 0.2 & 0.6 & 3.8 & 13.3 & 22.3 & 5.5 & 20.3 \\
\hline
\end{tabular}




\section{Section S4: Results}

\section{S4.1: Resource recovery efficiencies - MRF outputs and quality}

Table S15: Recyclable outputs contributing to the resource recovery rate for all scenarios.

\begin{tabular}{|c|c|c|c|c|c|c|c|c|c|c|c|}
\hline \multirow{2}{*}{ \# } & \multicolumn{2}{|c|}{ High quality } & \multicolumn{4}{|c|}{ Medium quality } & \multicolumn{5}{|c|}{ Low quality } \\
\hline & PET & HDPE & PET & HDPE & $P P+P S$ & Film & PET & HDPE & $P P+P S$ & Film & Mix \\
\hline 1 & 0.00 & 0.00 & 0.00 & 0.00 & 0.00 & 0.00 & 0.07 & 0.00 & 0.00 & 0.00 & 0.08 \\
\hline 2 & 0.00 & 0.00 & 0.11 & 0.00 & 0.00 & 0.00 & 0.00 & 0.03 & 0.00 & 0.00 & 0.00 \\
\hline 3 & 0.14 & 0.04 & 0.00 & 0.00 & 0.00 & 0.00 & 0.00 & 0.00 & 0.00 & 0.00 & 0.00 \\
\hline 4 & 0.00 & 0.00 & 0.00 & 0.00 & 0.00 & 0.00 & 0.00 & 0.00 & 0.00 & 0.00 & 0.00 \\
\hline 5 & 0.00 & 0.00 & 0.15 & 0.00 & 0.00 & 0.00 & 0.00 & 0.05 & 0.00 & 0.00 & 0.00 \\
\hline 6 & 0.20 & 0.06 & 0.00 & 0.00 & 0.00 & 0.00 & 0.00 & 0.00 & 0.00 & 0.00 & 0.00 \\
\hline 7 & 0.00 & 0.00 & 0.00 & 0.00 & 0.00 & 0.00 & 0.00 & 0.00 & 0.00 & 0.00 & 0.00 \\
\hline 8 & 0.00 & 0.00 & 0.00 & 0.00 & 0.00 & 0.00 & 0.00 & 0.00 & 0.00 & 0.00 & 0.00 \\
\hline 9 & 0.00 & 0.00 & 0.00 & 0.00 & 0.00 & 0.00 & 0.00 & 0.00 & 0.00 & 0.00 & 0.00 \\
\hline 10 & 0.00 & 0.00 & 0.11 & 0.00 & 0.00 & 0.00 & 0.00 & 0.03 & 0.00 & 0.00 & 0.00 \\
\hline 11 & 0.00 & 0.00 & 0.12 & 0.04 & 0.01 & 0.00 & 0.00 & 0.00 & 0.00 & 0.00 & 0.00 \\
\hline 12 & 0.00 & 0.00 & 0.12 & 0.04 & 0.01 & 0.00 & 0.00 & 0.00 & 0.00 & 0.00 & 0.00 \\
\hline 13 & 0.14 & 0.04 & 0.00 & 0.00 & 0.00 & 0.00 & 0.00 & 0.00 & 0.00 & 0.00 & 0.00 \\
\hline 14 & 0.15 & 0.05 & 0.00 & 0.00 & 0.02 & 0.00 & 0.00 & 0.00 & 0.00 & 0.00 & 0.00 \\
\hline 15 & 0.15 & 0.05 & 0.00 & 0.00 & 0.02 & 0.00 & 0.00 & 0.00 & 0.00 & 0.00 & 0.00 \\
\hline 16 & 0.00 & 0.00 & 0.00 & 0.00 & 0.00 & 0.00 & 0.00 & 0.00 & 0.00 & 0.00 & 0.00 \\
\hline 17 & 0.00 & 0.00 & 0.00 & 0.00 & 0.00 & 0.00 & 0.00 & 0.00 & 0.00 & 0.00 & 0.00 \\
\hline 18 & 0.00 & 0.00 & 0.00 & 0.00 & 0.00 & 0.00 & 0.00 & 0.00 & 0.00 & 0.00 & 0.00 \\
\hline 19 & 0.00 & 0.00 & 0.16 & 0.00 & 0.00 & 0.00 & 0.00 & 0.05 & 0.00 & 0.00 & 0.00 \\
\hline 20 & 0.00 & 0.00 & 0.00 & 0.00 & 0.03 & 0.00 & 0.17 & 0.06 & 0.00 & 0.00 & 0.00 \\
\hline 21 & 0.00 & 0.00 & 0.00 & 0.00 & 0.03 & 0.00 & 0.17 & 0.06 & 0.00 & 0.00 & 0.00 \\
\hline 22 & 0.20 & 0.06 & 0.00 & 0.00 & 0.00 & 0.00 & 0.00 & 0.00 & 0.00 & 0.00 & 0.00 \\
\hline 23 & 0.22 & 0.07 & 0.00 & 0.00 & 0.04 & 0.00 & 0.00 & 0.00 & 0.00 & 0.00 & 0.00 \\
\hline 24 & 0.22 & 0.07 & 0.00 & 0.00 & 0.04 & 0.00 & 0.00 & 0.00 & 0.01 & 0.07 & 0.00 \\
\hline 25 & 0.00 & 0.00 & 0.00 & 0.00 & 0.00 & 0.00 & 0.00 & 0.00 & 0.00 & 0.00 & 0.00 \\
\hline 26 & 0.00 & 0.00 & 0.00 & 0.00 & 0.00 & 0.00 & 0.00 & 0.00 & 0.00 & 0.00 & 0.00 \\
\hline 27 & 0.00 & 0.00 & 0.00 & 0.00 & 0.00 & 0.00 & 0.00 & 0.00 & 0.00 & 0.00 & 0.00 \\
\hline 28 & 0.00 & 0.00 & 0.00 & 0.00 & 0.00 & 0.00 & 0.12 & 0.04 & 0.00 & 0.00 & 0.00 \\
\hline 29 & 0.00 & 0.00 & 0.00 & 0.00 & 0.01 & 0.00 & 0.12 & 0.04 & 0.00 & 0.00 & 0.00 \\
\hline 30 & 0.00 & 0.00 & 0.00 & 0.00 & 0.01 & 0.05 & 0.12 & 0.04 & 0.00 & 0.00 & 0.00 \\
\hline 31 & 0.15 & 0.04 & 0.00 & 0.00 & 0.00 & 0.00 & 0.00 & 0.00 & 0.00 & 0.00 & 0.00 \\
\hline 32 & 0.16 & 0.05 & 0.00 & 0.00 & 0.02 & 0.00 & 0.00 & 0.00 & 0.00 & 0.00 & 0.00 \\
\hline 33 & 0.16 & 0.05 & 0.00 & 0.00 & 0.02 & 0.10 & 0.00 & 0.00 & 0.00 & 0.00 & 0.00 \\
\hline 34 & 0.00 & 0.00 & 0.00 & 0.00 & 0.00 & 0.00 & 0.00 & 0.00 & 0.00 & 0.00 & 0.00 \\
\hline 35 & 0.00 & 0.00 & 0.00 & 0.00 & 0.00 & 0.00 & 0.00 & 0.00 & 0.00 & 0.00 & 0.00 \\
\hline
\end{tabular}


2018 Journal of Industrial Ecology - www.wileyonlinelibrary.com/journal/iie

Table S15 (contin.): Recyclable outputs contributing to the resource recovery rate for all scenarios.

\begin{tabular}{|c|c|c|c|c|c|c|c|c|c|c|c|}
\hline \multirow{2}{*}{ \# } & \multicolumn{2}{|c|}{ High quality } & \multicolumn{4}{|c|}{ Medium quality } & \multicolumn{5}{|c|}{ Low quality } \\
\hline & PET & HDPE & PET & HDPE & $P P+P S$ & Film & PET & HDPE & $P P+P S$ & Film & Mix \\
\hline 36 & 0.00 & 0.00 & 0.00 & 0.00 & 0.00 & 0.00 & 0.00 & 0.00 & 0.00 & 0.00 & 0.00 \\
\hline 37 & 0.00 & 0.00 & 0.00 & 0.00 & 0.00 & 0.00 & 0.17 & 0.00 & 0.00 & 0.00 & 0.00 \\
\hline 38 & 0.00 & 0.00 & 0.00 & 0.00 & 0.03 & 0.00 & 0.18 & 0.06 & 0.00 & 0.00 & 0.00 \\
\hline 39 & 0.00 & 0.00 & 0.00 & 0.00 & 0.03 & 0.11 & 0.18 & 0.06 & 0.00 & 0.00 & 0.00 \\
\hline 40 & 0.21 & 0.06 & 0.00 & 0.00 & 0.00 & 0.00 & 0.00 & 0.00 & 0.00 & 0.00 & 0.00 \\
\hline 41 & 0.23 & 0.08 & 0.00 & 0.00 & 0.04 & 0.00 & 0.00 & 0.00 & 0.00 & 0.00 & 0.00 \\
\hline 42 & 0.23 & 0.08 & 0.00 & 0.00 & 0.04 & 0.19 & 0.00 & 0.00 & 0.00 & 0.00 & 0.00 \\
\hline 43 & 0.00 & 0.00 & 0.00 & 0.00 & 0.00 & 0.00 & 0.00 & 0.00 & 0.00 & 0.00 & 0.00 \\
\hline 44 & 0.00 & 0.00 & 0.00 & 0.00 & 0.00 & 0.00 & 0.00 & 0.00 & 0.00 & 0.00 & 0.00 \\
\hline 45 & 0.00 & 0.00 & 0.00 & 0.00 & 0.00 & 0.00 & 0.00 & 0.00 & 0.00 & 0.00 & 0.00 \\
\hline 46 & 0.00 & 0.00 & 0.11 & 0.00 & 0.00 & 0.00 & 0.00 & 0.03 & 0.00 & 0.00 & 0.00 \\
\hline 47 & 0.00 & 0.00 & 0.12 & 0.04 & 0.01 & 0.00 & 0.00 & 0.00 & 0.00 & 0.00 & 0.00 \\
\hline 48 & 0.00 & 0.00 & 0.12 & 0.04 & 0.01 & 0.00 & 0.00 & 0.00 & 0.00 & 0.00 & 0.00 \\
\hline 49 & 0.15 & 0.04 & 0.00 & 0.00 & 0.00 & 0.00 & 0.00 & 0.00 & 0.00 & 0.00 & 0.00 \\
\hline 50 & 0.15 & 0.05 & 0.00 & 0.00 & 0.02 & 0.00 & 0.00 & 0.00 & 0.00 & 0.00 & 0.00 \\
\hline 51 & 0.15 & 0.05 & 0.00 & 0.00 & 0.02 & 0.00 & 0.00 & 0.00 & 0.00 & 0.00 & 0.00 \\
\hline 52 & 0.00 & 0.00 & 0.00 & 0.00 & 0.00 & 0.00 & 0.00 & 0.00 & 0.00 & 0.00 & 0.00 \\
\hline 53 & 0.00 & 0.00 & 0.00 & 0.00 & 0.00 & 0.00 & 0.00 & 0.00 & 0.00 & 0.00 & 0.00 \\
\hline 54 & 0.00 & 0.00 & 0.00 & 0.00 & 0.00 & 0.00 & 0.00 & 0.00 & 0.00 & 0.00 & 0.00 \\
\hline 55 & 0.00 & 0.00 & 0.00 & 0.00 & 0.00 & 0.00 & 0.16 & 0.05 & 0.00 & 0.00 & 0.00 \\
\hline 56 & 0.00 & 0.00 & 0.17 & 0.00 & 0.03 & 0.00 & 0.00 & 0.06 & 0.00 & 0.00 & 0.00 \\
\hline 57 & 0.00 & 0.00 & 0.17 & 0.00 & 0.03 & 0.00 & 0.00 & 0.06 & 0.00 & 0.00 & 0.00 \\
\hline 58 & 0.20 & 0.06 & 0.00 & 0.00 & 0.00 & 0.00 & 0.00 & 0.00 & 0.00 & 0.00 & 0.00 \\
\hline 59 & 0.22 & 0.07 & 0.00 & 0.00 & 0.04 & 0.00 & 0.00 & 0.00 & 0.00 & 0.00 & 0.00 \\
\hline 60 & 0.22 & 0.07 & 0.00 & 0.00 & 0.04 & 0.00 & 0.00 & 0.00 & 0.01 & 0.07 & 0.00 \\
\hline 61 & 0.00 & 0.00 & 0.00 & 0.00 & 0.00 & 0.00 & 0.00 & 0.00 & 0.00 & 0.00 & 0.00 \\
\hline 62 & 0.00 & 0.00 & 0.00 & 0.00 & 0.00 & 0.00 & 0.00 & 0.00 & 0.00 & 0.00 & 0.00 \\
\hline 63 & 0.00 & 0.00 & 0.00 & 0.00 & 0.00 & 0.00 & 0.00 & 0.00 & 0.00 & 0.00 & 0.00 \\
\hline 64 & 0.00 & 0.00 & 0.00 & 0.00 & 0.00 & 0.00 & 0.12 & 0.04 & 0.00 & 0.00 & 0.00 \\
\hline 65 & 0.00 & 0.00 & 0.00 & 0.00 & 0.01 & 0.00 & 0.12 & 0.04 & 0.00 & 0.00 & 0.00 \\
\hline 66 & 0.00 & 0.00 & 0.00 & 0.00 & 0.01 & 0.05 & 0.12 & 0.04 & 0.00 & 0.00 & 0.00 \\
\hline 67 & 0.15 & 0.04 & 0.00 & 0.00 & 0.00 & 0.00 & 0.00 & 0.00 & 0.00 & 0.00 & 0.00 \\
\hline 68 & 0.16 & 0.05 & 0.00 & 0.00 & 0.02 & 0.00 & 0.00 & 0.00 & 0.00 & 0.00 & 0.00 \\
\hline 69 & 0.16 & 0.05 & 0.00 & 0.00 & 0.02 & 0.10 & 0.00 & 0.00 & 0.00 & 0.00 & 0.00 \\
\hline 70 & 0.00 & 0.00 & 0.00 & 0.00 & 0.00 & 0.00 & 0.00 & 0.00 & 0.00 & 0.00 & 0.00 \\
\hline 71 & 0.00 & 0.00 & 0.00 & 0.00 & 0.00 & 0.00 & 0.00 & 0.00 & 0.00 & 0.00 & 0.00 \\
\hline 72 & 0.00 & 0.00 & 0.00 & 0.00 & 0.00 & 0.00 & 0.00 & 0.00 & 0.00 & 0.00 & 0.00 \\
\hline 73 & 0.00 & 0.00 & 0.00 & 0.00 & 0.00 & 0.00 & 0.17 & 0.00 & 0.00 & 0.00 & 0.00 \\
\hline
\end{tabular}


2018 Journal of Industrial Ecology - www.wileyonlinelibrary.com/journal/jie

Table S15 (contin.): Recyclable outputs contributing to the resource recovery rate for all scenarios.

\begin{tabular}{l|cc|cccc|ccccc}
\hline \multirow{2}{*}{} & \multicolumn{3}{|c|}{ High quality } & \multicolumn{5}{|c|}{ Medium quality } & \multicolumn{5}{c}{ Low quality } \\
& PET & HDPE & PET & HDPE & PP+PS & Film & PET & HDPE & PP+PS & Film & Mix \\
\hline 74 & 0.00 & 0.00 & 0.00 & 0.00 & 0.03 & 0.00 & 0.18 & 0.06 & 0.00 & 0.00 & 0.00 \\
75 & 0.00 & 0.00 & 0.00 & 0.00 & 0.03 & 0.11 & 0.18 & 0.06 & 0.00 & 0.00 & 0.00 \\
76 & 0.21 & 0.06 & 0.00 & 0.00 & 0.00 & 0.00 & 0.00 & 0.00 & 0.00 & 0.00 & 0.00 \\
77 & 0.23 & 0.08 & 0.00 & 0.00 & 0.04 & 0.00 & 0.00 & 0.00 & 0.00 & 0.00 & 0.00 \\
78 & 0.23 & 0.08 & 0.00 & 0.00 & 0.01 & 0.19 & 0.00 & 0.00 & 0.00 & 0.00 & 0.00 \\
79 & 0.00 & 0.00 & 0.00 & 0.00 & 0.00 & 0.00 & 0.00 & 0.00 & 0.00 & 0.00 & 0.00 \\
80 & 0.00 & 0.00 & 0.00 & 0.00 & 0.00 & 0.00 & 0.00 & 0.00 & 0.00 & 0.00 & 0.00 \\
81 & 0.00 & 0.00 & 0.00 & 0.00 & 0.00 & 0.00 & 0.00 & 0.00 & 0.00 & 0.00 & 0.00 \\
82 & 0.00 & 0.00 & 0.00 & 0.00 & 0.05 & 0.00 & 0.00 & 0.08 & 0.00 & 0.00 & 0.00 \\
83 & 0.00 & 0.00 & 0.00 & 0.00 & 0.00 & 0.00 & 0.00 & 0.00 & 0.00 & 0.00 & 0.00 \\
84 & 0.00 & 0.00 & 0.00 & 0.00 & 0.05 & 0.18 & 0.00 & 0.08 & 0.00 & 0.00 & 0.00 \\
\hline
\end{tabular}

Table S16: Non-recyclable outputs contributing to the resource recovery rate for all scenarios.

\begin{tabular}{l|cccccc}
\hline$\#$ & PET & HDPE & PP & PS & Film & Mix \\
\hline 1 & 0.00 & 0.13 & 0.00 & 0.00 & 0.00 & 0.00 \\
2 & 0.00 & 0.00 & 0.00 & 0.00 & 0.00 & 0.32 \\
3 & 0.00 & 0.00 & 0.00 & 0.00 & 0.00 & 0.23 \\
4 & 0.41 & 0.13 & 0.00 & 0.00 & 0.00 & 0.46 \\
5 & 0.00 & 0.00 & 0.00 & 0.00 & 0.00 & 0.36 \\
6 & 0.00 & 0.00 & 0.00 & 0.00 & 0.00 & 0.29 \\
7 & 0.40 & 0.13 & 0.00 & 0.00 & 0.00 & 0.47 \\
8 & 0.35 & 0.11 & 0.03 & 0.00 & 0.00 & 0.51 \\
9 & 0.35 & 0.11 & 0.03 & 0.01 & 0.02 & 0.48 \\
10 & 0.00 & 0.00 & 0.00 & 0.00 & 0.00 & 0.37 \\
11 & 0.00 & 0.00 & 0.00 & 0.00 & 0.00 & 0.33 \\
12 & 0.00 & 0.00 & 0.00 & 0.01 & 0.08 & 0.24 \\
13 & 0.00 & 0.00 & 0.00 & 0.00 & 0.00 & 0.29 \\
14 & 0.00 & 0.00 & 0.00 & 0.00 & 0.00 & 0.18 \\
15 & 0.00 & 0.00 & 0.00 & 0.01 & 0.12 & 0.05 \\
16 & 0.40 & 0.13 & 0.00 & 0.00 & 0.00 & 0.48 \\
17 & 0.33 & 0.10 & 0.04 & 0.00 & 0.00 & 0.53 \\
18 & 0.33 & 0.10 & 0.04 & 0.01 & 0.02 & 0.49 \\
19 & 0.00 & 0.00 & 0.00 & 0.00 & 0.00 & 0.41 \\
20 & 0.00 & 0.00 & 0.00 & 0.00 & 0.00 & 0.36 \\
21 & 0.00 & 0.00 & 0.00 & 0.01 & 0.10 & 0.25 \\
22 & 0.00 & 0.00 & 0.00 & 0.00 & 0.00 & 0.36 \\
23 & 0.00 & 0.00 & 0.00 & 0.00 & 0.00 & 0.22 \\
\hline
\end{tabular}


Table S16 (contin.): Non-recyclable outputs contributing to the resource recovery rate for all scenarios.

\begin{tabular}{|c|c|c|c|c|c|c|}
\hline$\#$ & PET & HDPE & PP & PS & Film & Mix \\
\hline 24 & 0.00 & 0.00 & 0.00 & 0.00 & 0.00 & 0.05 \\
\hline 25 & 0.39 & 0.12 & 0.00 & 0.00 & 0.00 & 0.49 \\
\hline 26 & 0.32 & 0.10 & 0.03 & 0.00 & 0.00 & 0.56 \\
\hline 27 & 0.32 & 0.10 & 0.03 & 0.01 & 0.04 & 0.51 \\
\hline 28 & 0.00 & 0.00 & 0.00 & 0.00 & 0.00 & 0.45 \\
\hline 29 & 0.00 & 0.00 & 0.00 & 0.00 & 0.00 & 0.43 \\
\hline 30 & 0.00 & 0.00 & 0.00 & 0.01 & 0.00 & 0.25 \\
\hline 31 & 0.00 & 0.00 & 0.00 & 0.00 & 0.00 & 0.43 \\
\hline 32 & 0.00 & 0.00 & 0.00 & 0.00 & 0.00 & 0.34 \\
\hline 33 & 0.00 & 0.00 & 0.00 & 0.01 & 0.00 & 0.04 \\
\hline 34 & 0.38 & 0.12 & 0.00 & 0.00 & 0.00 & 0.50 \\
\hline 35 & 0.29 & 0.09 & 0.04 & 0.00 & 0.00 & 0.58 \\
\hline 36 & 0.29 & 0.09 & 0.04 & 0.01 & 0.05 & 0.53 \\
\hline 37 & 0.00 & 0.11 & 0.00 & 0.00 & 0.00 & 0.51 \\
\hline 38 & 0.00 & 0.00 & 0.00 & 0.00 & 0.00 & 0.48 \\
\hline 39 & 0.00 & 0.00 & 0.00 & 0.01 & 0.00 & 0.26 \\
\hline 40 & 0.00 & 0.00 & 0.00 & 0.00 & 0.00 & 0.51 \\
\hline 41 & 0.00 & 0.00 & 0.00 & 0.00 & 0.00 & 0.40 \\
\hline 42 & 0.00 & 0.00 & 0.00 & 0.01 & 0.00 & 0.05 \\
\hline 43 & 0.38 & 0.12 & 0.00 & 0.00 & 0.00 & 0.50 \\
\hline 44 & 0.33 & 0.10 & 0.03 & 0.00 & 0.00 & 0.53 \\
\hline 45 & 0.33 & 0.10 & 0.03 & 0.01 & 0.02 & 0.51 \\
\hline 46 & 0.00 & 0.00 & 0.00 & 0.00 & 0.00 & 0.39 \\
\hline 47 & 0.00 & 0.00 & 0.00 & 0.00 & 0.00 & 0.35 \\
\hline 48 & 0.00 & 0.00 & 0.00 & 0.01 & 0.08 & 0.26 \\
\hline 49 & 0.00 & 0.00 & 0.00 & 0.00 & 0.00 & 0.30 \\
\hline 50 & 0.00 & 0.00 & 0.00 & 0.00 & 0.00 & 0.19 \\
\hline 51 & 0.00 & 0.00 & 0.00 & 0.01 & 0.12 & 0.05 \\
\hline 52 & 0.36 & 0.11 & 0.00 & 0.00 & 0.00 & 0.53 \\
\hline 53 & 0.31 & 0.10 & 0.04 & 0.00 & 0.00 & 0.56 \\
\hline 54 & 0.31 & 0.10 & 0.04 & 0.01 & 0.02 & 0.53 \\
\hline 55 & 0.00 & 0.00 & 0.00 & 0.00 & 0.00 & 0.44 \\
\hline 56 & 0.00 & 0.00 & 0.00 & 0.00 & 0.00 & 0.39 \\
\hline 57 & 0.00 & 0.00 & 0.00 & 0.01 & 0.09 & 0.28 \\
\hline 58 & 0.00 & 0.00 & 0.00 & 0.00 & 0.00 & 0.37 \\
\hline 59 & 0.00 & 0.00 & 0.00 & 0.00 & 0.00 & 0.23 \\
\hline 60 & 0.00 & 0.00 & 0.00 & 0.00 & 0.00 & 0.06 \\
\hline 61 & 0.34 & 0.11 & 0.00 & 0.00 & 0.00 & 0.55 \\
\hline 62 & 0.29 & 0.09 & 0.03 & 0.00 & 0.00 & 0.59 \\
\hline
\end{tabular}


2018 Journal of Industrial Ecology - www.wileyonlinelibrary.com/journal/jie

Table S16 (contin.): Non-recyclable outputs contributing to the resource recovery rate for all scenarios.

\begin{tabular}{l|cccccc}
\hline$\#$ & PET & HDPE & PP & PS & Film & Mix \\
\hline 63 & 0.29 & 0.09 & 0.03 & 0.01 & 0.03 & 0.55 \\
64 & 0.00 & 0.00 & 0.00 & 0.00 & 0.00 & 0.48 \\
65 & 0.00 & 0.00 & 0.00 & 0.00 & 0.00 & 0.46 \\
66 & 0.00 & 0.00 & 0.00 & 0.01 & 0.00 & 0.29 \\
67 & 0.00 & 0.00 & 0.00 & 0.00 & 0.00 & 0.44 \\
68 & 0.00 & 0.00 & 0.00 & 0.00 & 0.00 & 0.35 \\
69 & 0.00 & 0.00 & 0.00 & 0.01 & 0.00 & 0.06 \\
70 & 0.32 & 0.10 & 0.00 & 0.00 & 0.00 & 0.58 \\
71 & 0.26 & 0.08 & 0.03 & 0.00 & 0.00 & 0.62 \\
72 & 0.26 & 0.08 & 0.03 & 0.01 & 0.04 & 0.58 \\
73 & 0.00 & 0.11 & 0.00 & 0.00 & 0.00 & 0.54 \\
74 & 0.00 & 0.00 & 0.00 & 0.00 & 0.00 & 0.51 \\
75 & 0.00 & 0.00 & 0.00 & 0.01 & 0.00 & 0.31 \\
76 & 0.00 & 0.00 & 0.00 & 0.00 & 0.00 & 0.52 \\
77 & 0.00 & 0.00 & 0.00 & 0.00 & 0.00 & 0.41 \\
78 & 0.00 & 0.00 & 0.00 & 0.01 & 0.00 & 0.06 \\
79 & 0.26 & 0.08 & 0.00 & 0.00 & 0.00 & 0.66 \\
80 & 0.28 & 0.08 & 0.00 & 0.00 & 0.00 & 0.64 \\
81 & 0.21 & 0.06 & 0.03 & 0.00 & 0.00 & 0.70 \\
82 & 0.26 & 0.00 & 0.00 & 0.00 & 0.00 & 0.60 \\
83 & 0.21 & 0.06 & 0.03 & 0.01 & 0.04 & 0.65 \\
84 & 0.26 & 0.00 & 0.00 & 0.01 & 0.00 & 0.40 \\
\hline
\end{tabular}




\section{References}

Association of postconsumer plastic recyclers (APR), 2013a. Model bale specifications: MRF mix film [online document]. http://www. plasticsrecycling.org/images/pdf/Markets/MRF Curbside Film.pdf [6 September 2018].

Association of postconsumer plastic recyclers (APR), 2013b. Model postconsumer bale specifications. PET Thermoforms [online document]. http://www.plasticsrecycling.org/images/pdf/Markets/PET Thermoforms.pdf [6 September 2018].

Association of postconsumer plastic recyclers (APR), 2016a. Model bale specifications - Polypropylene [online document]. http://www.plasticsrecycling.org/images/pdf/PE PP Resins/For-MRFS-Reclaimers/PP Model Bale Spec.pdf [15 August 2016].

Association of postconsumer plastic recyclers (APR), 2016b. Model bale specifications - HDPE injection bulky rigids [online document]. http://www.plasticsrecycling.org/images/pdf/Markets/HDPE Injection Bulky Rigid Plastics.pdf [6 September 2018].

Association of postconsumer plastic recyclers (APR), 2016c. Unpigmented (natural) HDPE Bottles - Model bale specifications and gradings [online document]. http://www.plasticsrecycling.org/images/pdf/PE PP Resins/Bale Specs/Natural Graded HDPE.pdf [15 August 2016].

Association of postconsumer plastic recyclers (APR), 2016d. Model bale specification - All rigid plastic [online document]. http://www.plasticsrecycling.org/images/pdf/Markets/1 7 Bottles and All Rigid Plastics.pdf [6 September 2018].

Ballesteros-Gómez, A., Brandsma, S.H., de Boer, J., Leonards, P.E.G., 2014. Direct probe at-mospheric pressure photoionization / atmospheric pressure chemical ionization high-resolution mass spectrometry for fast screening of flame retardants and plasticizers in products and waste. Analytical and Bioanalytical Chemistry. http://doi.org/10.1007/s00216-014-7636-8

Baytech Plastics, 2015. Plastic Toy Manufactures: USA Firms Balance Cost, Innovation, And Quality. [website]. http://www.baytechplastics.com/plastic-toymanufacturers-usa-firms-balance-cost-innovation-and-quality [6 September 2018].

Biffa Polymers, 2016. Postconsumer PP rich baled plastics. Incoming quality specification. [online document]. http://resourceassociation.com/sites/all/themes/ra resource/qspecs/plastics/Biffa's\%20Mixed\%20plastics\%20Plant\%20\%20PP\%20\%20bale\%20spec.pdf [6 September 2018].

Bing, X., Bloemhof-Ruwaard, J.M., van der Vorst, J.G.A.J., 2014. Sustainable reverse logistics network design for household plastic waste. Flexible Services and Manufacturing Journal. http://doi.org/10.1007/s10696-012-9149-0

Cappadona, B., 2015. Residential Recycling Top Contaminants [online lecture]. US Environmental Protection Agency, Sustainable Materials Management Webinar, December 172015 [5:00-25:22]. https://www.youtube.com/watch?v=-rS2F9r IGE\&feature=youtu.be [6 September 2018]. 
Cimpan, C., Maul, A., Jansen, M., Thomas, P., Henrik, W., 2015. Central sorting and recovery of MSW recyclable materials: A review of technological state-ofthe-art, cases, practice and implications for materials recycling. Journal of Environmental Management. http://doi.org/10.1016/j.jenvman.2015.03.025

Clemen, J., 2014. Plastikstatistik [slide show]. Plastindustrien - brancheforeningen for danske plastvirksomheder.

CLR (Closed loop recycling), 2016a. Specification sheet for pre-sorted HDPE bales [online doc-ument]. http://resourceassociation.com/sites/all/themes/ra resource/qspecs/plastics/Closed\%20Loop\%20011-01\%20Incoming\%20pre\%20sorted\%20HDPE\%20bales.pdf [6 September 2018].

CLR (Closed loop recycling), 2016b. Specification sheet for pre-sorted PET bales [online docu-ment]. http://resourceassociation.com/sites/all/themes/ra resource/qspecs/plastics/Closed\%20Loop\%20012-01\%20Incoming\%20pre\%20sorted\%20PET\%20bales.pdf [6 September 2018].

Cornell, D. 2016. Personal communication. Association of plastic recyclers.

Daugaard, A. E., 2015. Personal communication. Associate professor at the department of chemical and biochemical engineering, technical university of Denmark.

Der Grüne Punkt (DKR), 2012a. Product Specification 05/2012 Fraction-No. 324 - Polypropylene [online document]. Can be accessed via: http://www.gruenerpunkt.de/en/download.html [6 September 2018]

Der Grüne Punkt (DKR), 2012b. Product Specification 05/2012 Fraction-No. 331 - Polystyrene [online document]. Can be accessed via: http://www.gruenerpunkt.de/en/download.html [6 September 2018]

Der Grüne Punkt (DKR), 2012c. Product Specification 05/2012 Fraction-No. 329 - Polyethylene [online document]. Can be accessed via: http://www.gruenerpunkt.de/en/download.html [6 September 2018]

Der Grüne Punkt (DKR), 2012d. Product Specification 05/2012 Fraction-No. 352 - New mixed plastic [online document]. Can be accessed via: http://www.gruener-punkt.de/en/download.html [6 September 2018]

Der Grüne Punkt (DKR), 2014a. Product Specification 08/2014 Fraction-No. 325 - PET Bottles, transparent [online document]. Can be accessed via: http://www.gruener-punkt.de/en/download.html [6 September 2018]

Der Grüne Punkt (DKR), 2014b. Product Specification 08/2014 Fraction-No. 3283 - Mixed PET 50/50 [online document]. Can be accessed via:

http://www.gruener-punkt.de/en/download.html [6 September 2018]

Der Grüne Punkt (DKR), 2014c. Product Specification 08/2014 Fraction-No. 3101 - Plastic films [online document]. Can be accessed via: http://www.gruenerpunkt.de/en/download.html [6 September 2018]

Dvorak, R., Evans, R., Kosior, E., 2009. Commercial scale mixed plastics recycling. UK: WRAP.

Edjabou, M. E., Jensen, M. B., Götze, R., Pivnenko, K., Petersen, C., Scheutz, C., Astrup, T. F., 2015. Municipal solid waste composition: Sampling methodology, statistical analyses, and case study evaluation. Waste management. http://doi.org/10.1016/j.wasman.2014.11.009 
EC (European Commission), 2013. Toy Safety Directive 2009/48/EC. An explanatory guidance document of 13 December 2013, Rev 1.7. Brussels: European Commission.

Enviros, 2009. MRF quality assessment study - material quality assessment of municipal MRFs within the UK. WRAP, MRF011.

Ernst, T., Popp, R., van Eldik, R., 2000. Quantification of heavy metals for the recycling of waste plastics from electrotechnical applications. Talanta. http://doi.org/10.1016/S0039-9140(00)00491-4

EU (European Union), 2006. Regulation (EC) No 1907/2006 of the European Parliament and of the Council concerning the Registration, Evaluation, Authorisation and Restriction of Chemicals (REACH), establishing a European Chemicals Agency, amending Directive 1999/45/EC and repealing Council Regulation (EEC) No 793/93 and Commission Regulation (EC) No 1488/94 as well as Council Direc- tive 76/769/EEC and Commission Directives 91/155/EEC, 93/67/EEC, 93/105/EC and 2000/21/EC of 18 December 2006. Official Journal of the European Union. L136, 3-280.

EU (European Union), 2007. Council directive 93/42/EEC concerning medical devices of 14 June 1993. Amended 5 times, lastly by Directive 2007/47/EC of the European Parliament and of the Council of 5 September 2007.

EU (European Union), 2011a. Regulation (EU) No 305/2011 of the European Parliament and the Council of 9 March 2011 laying down harmonised conditions for the marketing of construction products and repealing Council Directive 89/106/ECC. Official Journal of the European Union. L88, 5-43.

EU (European Union), 2011b. Commission Regulation (EU) No 10/2011 on plastic materials and articles intended to come into contact with food of 14 January 2011. Official Journal of the European Union. L12, 1-89.

EU (European Union), 2011c. Directive 2011/65/EU of the European parliament and of the council of the restriction of the use of certain hazardous substances in electrical and electronic equipment (recast) of 8 June 2011. Official Journal of the European Union. L174, 88-110.

Eurostat, 2015. Waste generation [database]. http://appsso.eurostat.ec.europa.eu/nui/submitViewTableAction.do [13 April 2015].

Feil, A., Pretz, T., Jansen, M., van Velzen, E., 2016. Separate collection of plastic waste, better than technical sorting from municipal solid waste? Waste Management and Research. http://doi.org/10.1177/0734242X16654978

Goodman, T., 2006. SINGLE-STREAM AND DUAL-STREAM RECYCLING : COMPARATIVE IMPACTS OF COMMINGLED RECYCLABLES PROCESSING. Minnesota: Minnesota Pollution Control Agency.

Haupt, M., Vadenbo, C., Hellweg, S., Do we have the right performance indicators for the circular economy? - Insight into the Swiss waste management system. Journal of Industrial Ecology: http://doi.org/10.1111/jiec.12506

Heinzel, J.V., Larsen, C.S., Tønning, K., Malmgren-Hansen, B., Nilsson, N.H., 2015. Mekanisk sortering af plastaffald fra husholdninger (Mechanical sorting of plastic waste from households) [report]. Copenhagen: Danish Environmental Protection Agency. 
Huber, M., Franz, R., 1997. Identification of Migratable Substances in Recycled High Density Polyethylene Collected from Household Waste. Journal of High Resolution Chromatog-raphy, vol. 20(8), pp. 427-430.

Jansen, M., Fiel, A., Pretz, T., 2012. Recovery of Plastics from Household Waste by Mechanical Separation. [report]

LRS consultancy, 2015. WRAP Plastics Compositional Analysis at MRFs [online report]. Available at: http://www.wrap.org.uk/sites/files/wrap/WRAP\%20Plastics\%20Compositional\%20Analysis\%20FINAL\%20v2b May\%201\%202015 0.pdf [11 August 2016].

Luijsterburg, B., Goossens, H., 2014. Assessment of plastic packaging waste: Material origin, methods and properties. Resource, Conservation and Recycling, vol. 85, pp. 88-97. DOI: 10.1016/j.resconrec.2013.10.010.

Moreau, T., 2015. Managing "recycling" contamination - A public facility perspective. Available at: https://www.youtube.com/watch?v=-rS2F9r_IGE\&feature=youtu.be [23:00-48:30 - 20 June 2018].

Petersen, C., Manokaran, S., 2013. Restaffald I Haderslev, Sønderborg og Åbenrå Kommune - mængde og sammensætning af dagrenovation fra forskellige boligtyper med forskellige af-faldsordninger. Econet AS.

Petersen, C., Mayland, C., 2015. Genanvendeligt affald i indsamlet dagrenovation (Recyclable waste in collected residual household waste) [report]. Danish Environmental Protection Agency.

Petersen, C., Kaysen, O., Edjabou, V., Manokaren, S., Tønning, K., Hansen, T., 2012. Kortlægning af dagrenovation i enfamilieboliger [Characterization of residual waste in single family houses]. Danish Environmental Protection Agency.

Petersen, C., Kaysen, O., Manokaren, S., Tønning, K., Hansen, T., 2014. Kortlægning af dag-renovation i Danmark [Characterization of residual waste in Denmark]. Danish Environ-mental Protection Agency.

Pivnenko, K., Eriksen, M.K., Martín-Fernández, J.A., Eriksen, E., Astrup, T.F., 2016. Recycling of plastic waste: Presence of phthalates in plastics from households and industry. Waste management. http://doi.org10.1111/jiec.12506

PlasticsEurope, 2013. Automotive - The world moves with plastics [online brochure]. file://env-tsprofile.win.dtu.dk/TSProfiles/maker/Downloads/Automotive Flyer 2013.pdf [6 September 2018].

PlasticsEurope, EPRO (European Association of Plastic Recycling and Recovery Organisations) 2016. Plastics - the Facts 2016. An analysis of European plastics production, demand and waste data https://www.plasticseurope.org/application/files/4315/1310/4805/plastic-the-fact-2016.pdf [6 September 2018]

Riber, C., Petersen, C., Christensen, T.H., 2009. Chemical composition of material fractions in Danish household waste. Waste management. http://doi.org/10.1016/j.wasman.2008.09.013

Rigamonti L., Grosso, M., Møller, J., Martinez Sanchez, V., Magnani, S., Christensen T. H., 2014. Environmental evaluation of plastic waste management scenarios. Resources, Conservation and Recycling.

http://doi.org/10.1016/j.resconrec.2013.12.012 
ReQIP (Recycling Quality Information Point), 2014. Contamination value chart packaging [website]. http://www.resourceassociation.com/reqip-contaminationvalue-chart [6 September 2018].

RRS (Resource Recycling Systems), 2015. MRF material flow study - Final report 2015. Ann Arbor: Resource Recycling Systems.

Safe Toys, 2015. Appendix C - Directive 2009/48/EC. First Substances included: BPA, TDEP, TDCP, TCPP [online document]. http://www.icqglobal.com/eng/wp-content/uploads/2014/07/IISG AppendixC ENG1.pdf [6 September 2018].

Shonfield, P., 2008. LCA of management options for mixed waste plastics [online report]. WRAP, MDP017.

Spendelow, P., 2011. Composition of Commingled Recyclables Before and After Processing [report]. State of Oregon: Department of environmental quality.

Stubenschrott, V., 2016. flexible packaging - food : non food approval [personal e-mail correspondence]. Constantia Flexibles Group $\mathrm{GmbH}$, Corporate development. Received 28 November 2016.

TIE (Toy Industries of Europe), 2012. The European Toy Industry: Facts and Figures. https://www.toyindustries.eu/ [6 September 2018]

van der Harst, E., Potting, J., Kroeze, C., 2016. Comparison of different methods to include re-cycling in LCAs of aluminium cans and disposable polystyrene cups. Waste management. http://doi.org/10.1016/j.wasman.2015.09.027

van Velzen, U.T., Bos-Brouwers, H., Groot, J., Bing, X., Jansen, M., Luijsterburg, B., 2013. Scenarios study on post-consumer plastic packaging waste recycling. Wageningen: Wageningen UR Food \& Biobased Research, 1408.

Villanueva, A., Eder, P., 2014. End-of-Waste Criteria for waste plastic for conversion. Luxembourg: Publications Office of the European Union.

Whitt, M., Vorst, K., Brown, W., Baker, S., Gorman, L., 2012. Survey of heavy metal contami-nation in recycled polyethylene terephthalate used for food packaging. Journal of Plastic Film and Sheeting. http://doi.org/10.1177/8756087912467028

Ærenlund, L., 2016. Fra husholdningsplast til affaldssorteringssystem [slide show]. Contribution to DAKOFA conference on bio- and plastic waste 05 April 2016, Copenhagen. 